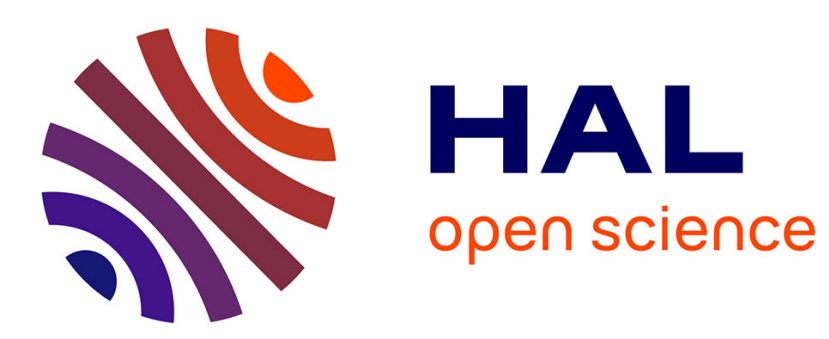

\title{
Morphological bilateral filtering
}

Jesus Angulo

\section{To cite this version:}

Jesus Angulo. Morphological bilateral filtering. SIAM Journal on Imaging Sciences, 2013, 6 (3), pp.1790-1822. 10.1137/110844258 . hal-00789160v1

\section{HAL Id: hal-00789160 \\ https://hal-mines-paristech.archives-ouvertes.fr/hal-00789160v1}

Submitted on 16 Feb 2013 (v1), last revised 26 Oct 2013 (v2)

HAL is a multi-disciplinary open access archive for the deposit and dissemination of scientific research documents, whether they are published or not. The documents may come from teaching and research institutions in France or abroad, or from public or private research centers.
L'archive ouverte pluridisciplinaire HAL, est destinée au dépôt et à la diffusion de documents scientifiques de niveau recherche, publiés ou non, émanant des établissements d'enseignement et de recherche français ou étrangers, des laboratoires publics ou privés. 


\title{
MORPHOLOGICAL BILATERAL FILTERING*
}

\author{
JESÚS ANGULO ${ }^{\dagger}$
}

\begin{abstract}
A current challenging topic in mathematical morphology is the construction of locally adaptive operators; i.e., structuring functions are dependent on the input image itself at each position. Development of spatially-variant filtering is well established in the theory and practice of Gaussian filtering. The aim of the first part of the paper is to study how to generalize these convolutionbased approaches in order to introduce adaptive nonlinear filters which asymptotically correspond to spatially-variant morphological dilation and erosion. In particular, starting from the bilateral filtering framework and using the notion counter-harmonic mean, our goal is to propose a new low complexity approach to define spatially-variant bilateral structuring functions. Then, in the second part of the paper, an original formulation of spatially-variant flat morphological filters is proposed, where the adaptive structuring elements are obtained by thresholding the bilateral structuring functions. The methodological results of the paper are illustrated with various comparative examples.
\end{abstract}

Key words. bilateral filtering, counter-harmonic mean, adaptive mathematical morphology, spatially variant structuring functions

\section{AMS subject classifications.}

1. Introduction. Mathematical morphology is a well-known nonlinear image processing methodology based on the application of lattice theory to spatial structures [46] [25]. Let $E$ be the Euclidean $\mathbb{R}^{d}$ or discrete space $\mathbb{Z}^{d}$ (support space) and let $\mathcal{T}$ be a set of grey-levels (space of values). It is assumed that $\mathcal{T}=\overline{\mathbb{R}}=\mathbb{R} \cup\{-\infty,+\infty\}$. A grey-level image is represented by a function $f: E \rightarrow \mathcal{T}, f \in \mathcal{F}(E, \mathcal{T})$, i.e., $f$ maps each pixel $\mathbf{x} \in E$ into a grey-level value $t \in \mathcal{T}: t=f(\mathbf{x})$. Given a grey-level image, the two basic morphological mappings $\mathcal{F}(E, \mathcal{T}) \rightarrow \mathcal{F}(E, \mathcal{T})$ are the dilation and the erosion given respectively by

$$
\delta_{b}(f)(\mathbf{x})=\sup _{\mathbf{y} \in E}(f(\mathbf{x}-\mathbf{y})+b(\mathbf{y}))=\sup _{\mathbf{y} \in E}(f(\mathbf{y})+b(\mathbf{y}-\mathbf{x}))
$$

and

$$
\varepsilon_{b}(f)(\mathbf{x})=\inf _{\mathbf{y} \in E}(f(\mathbf{x}+\mathbf{y})-b(\mathbf{y}))=\inf _{\mathbf{y} \in E}(f(\mathbf{y})-b(\mathbf{y}+\mathbf{x}))
$$

where $b \in \mathcal{F}(E, \mathcal{T})$ is the structuring function which determines the effect of the operator. If the support space of function $b$ is $\operatorname{supp}(b) \subset E$, i.e., a "window" smaller than the image domain, the two forms of equations (1.1) and (1.2) are valid if $b$ is extended to domain $E$ by putting $-\infty$ outside its bounded support. The other morphological operators, such as the opening and the closing, are obtained as products of dilation/erosion. The most commonly studied framework, which additionally presents better properties of invariance, is based on flat structuring functions, called structuring elements. More precisely, let $B$ be a Boolean set defined at the origin, i.e., $B \subseteq E$ or $B \in \mathcal{P}(E)$, which defines the "shape" of the structuring element, the associated structuring function is given by $b(\mathbf{x})=0$ if $\mathbf{x} \in B$ and $b(\mathbf{x})=-\infty$ if $\mathbf{x} \in B^{c}$

${ }^{*}$ This paper is an extended and improved version of a conference paper: J. Angulo. "Morphological bilateral filtering and spatially-variant adaptive structuring functions". In Proc. of ISMM'11 (2011 International Symposium on Mathematical Morphology), LNCS 6671, Springer-Verlag Berlin Heidelberg, p. 212-223, Intra (Lake Maggiore), Italy, July 2011.

†CMM-Centre de Morphologie Mathématique, Mathématiques et Systèmes, MINES Paristech; 35, rue Saint-Honoré, 77305 Fontainebleau cedex - France (jesus.angulo@mines-paristech.fr). 
(where $B^{c}$ is the complement set of $B$ ). Hence, the flat dilation and flat erosion can be computed respectively by the moving local maxima and minima filters, i.e., $\delta_{B}(f)(\mathbf{x})=\sup _{\mathbf{y} \in B}(f(\mathbf{x}-\mathbf{y}))$ and $\varepsilon_{B}(f)(\mathbf{x})=\inf _{\mathbf{y} \in B}(f(\mathbf{x}+\mathbf{y}))$, where the shape of the structuring element $B$ determines the effect of the operator.

Original formulation of dilation and erosion for grey-level images [45, 51], as well as the other morphological operators was translation invariant in the space and the intensity. Later, mathematical morphology was formulated in the framework of complete lattices [46, 25], which leads to a general case of dilation and adjoint erosion compatible with spatially-variant operators. Nevertheless, most of current implementations and classical applications studied in the literature are based on morphological operators which are translation invariant in the space and in the intensity [50], i.e., the same structuring function $b(\mathbf{x})$ (or structuring element $B$ ) is considered for each point $\mathbf{x}$ of the image. A current challenging topic in mathematical morphology is the construction of appropriate adaptive operators; i.e., structuring functions become dependent on the position or on the input image itself. In previous works, the adaptive operators are based on two main approaches. On the one hand, a variability on $E$ : spatially variable shape of structuring functions according to i) the position in the image $[4,16]$, ii) the local regularity $[36,23]$, iii) the local orientation [55]. On the other hand, a variability on $\mathcal{T}$ : variable size of structuring functions according to the local intensity or contrast $[54,39]$. For a recent overview on the state-the-art on adaptive morphology, the interested reader is invited to the paper [40]. Another recent study [43] is very interesting for understanding the theoretical limitations of input-adaptive morphological operators.

Aim of the paper. Development of locally adaptive filtering is well established in the theory and practice of Gaussian filtering. The aim of this paper is to study how to generalize these convolution based approaches in order to introduce adaptive nonlinear filters which asymptotically correspond to spatially-variant morphological dilation and erosion. In particular, starting from the bilateral filtering and using the notion of counter-harmonic mean, our goal is to propose a new low complexity approach to define spatially-variant structuring functions. After thresholding, adaptive structuring elements are obtained which can be used to compute spatially-variant dilation, erosion, opening and closing.

Related work. Bilateral filtering [52] is a locally adaptive Gaussian convolution technique to smooth images while preserving edges, where the Gaussian coefficients at a point are weighted by the intensity distance between its neighbors. Its formulation is quite simple and requires only two easily tunable parameters: the size and the contrast of the image features to preserve. A recent systematic study on the theory and applications of bilateral filtering can be found in [42]. As it was shown in [18, 19], bilateral filtering is strongly related to weighted least squares filtering [33], robust estimation filtering $[62,5]$ and anisotropic diffusion $[56,6]$ and Beltrami flow-based geometric diffusion $[47,30,48]$. In particular, bilateral filtering is a discrete filter equivalent asymptotically to Perona and Malik PDE equation [41, 15]. Another generalization of bilateral filtering is the spatial-tonal normalized convolution proposed in [8], which showed also the equivalence with local mode filtering. The idea of locally structure-adaptive convolution was also studied in [61] where an anisotropic Gaussian kernel (i.e., ellipsoid kernel) was defined by computing locally the structure orientation and an anisotropy parameter (i.e., Gaussian curvature). An optimized algorithm was later proposed for a fast implementation [21]. 
Bilateral filters are also related to the Lee's $\sigma$-filter [35], which only considers averaging in neighborhoods of pixels having similar intensities to the central pixel, but without including a spatial distance term. Another approach proposed also by Lee [34] to avoid the blurring effect of the spatial filtering consists in using a statistical (optimal) correction. Theoretical and empirical comparison of bilateral filtering and $\sigma$-filter was considered in [11], and they conclude that the bilateral filter performs a better denoising than Lee's filters. Non-local means (NL-means) [11], recently introduced in the continuity of the bilateral filter, is an image denoising process based on non-local averaging of all the pixels in an image. More precisely, the amount of weighting for a pixel in NL-means is based on the degree of similarity between a small patch centered around that pixel and the small patch centered around the pixel being denoised.

The construction of locally adaptive edge-preserving structuring elements has been previously considered in the literature of mathematical morphology. The notion of generalized geodesy [49] was proposed to introduce locally adaptive geodesic neighborhoods. Morphological amoebas [36] were proposed as a flexible discrete approach to compute locally adaptive structuring elements. Morphological amoebas have been basically considered for median filtering. In a recent study [59], it has been established an interesting correspondence between the iterated amoeba median filtering and the PDE of self-snakes. More recently, it has been introduced in [23] a framework to compute adaptive kernels using geodesic distances which generalizes the metric of morphological amoebas and adaptive geodesy. But it is exclusively used for averaging edge-preserving smoothing. We should remark that the adaptive neighborhoods associated to distance propagation in amoebas, or in generalized geodesy, involve a relatively high computational complexity.

We propose in this study an original formulation of adaptive morphological filters, with both volumetric and flat structuring functions, based on a nonlinearization of the bilateral filtering. The associated algorithms takes advantage of the low computational complexity of bilateral filtering. The starting point of the adopted approach is the notion of counter-harmonic mean [12]. In fact, the idea of using the counterharmonic mean to construct robust morphological-like operators, without the notions of supremum and infimum, was proposed in [53]. We have recently used in [2] the counter-harmonic framework to generalize image diffusion in order to introduce iterative nonlinear filters which effects mimic morphological dilation and erosion.

Paper organization. This paper is an extended and improved version of a conference contribution [3]. In particular, in the present manuscript, a complete proof of the main result is given as well as more details on the implementation of present operators. In addition, a better analysis of the behavior of the operators and more illustrative examples are included.

The outline of the paper is as follows. In next Section is reviewed, on the one hand, the notion of counter-harmonic mean filter and its appropriateness to approximate flat dilation/erosion. On the other hand, the principles of bilateral filtering are also reminded in Section 2. Section 3 introduces the proposed counter-harmonic generalization of bilateral filtering, including the limit relationships with various families of locally adaptive structuring functions. Generalization of these results to NL-means is also initiated. Section 4 discusses the construction of flat spatially variant morphology using thresholded adaptive structuring functions in the framework of morphological operators formulated by upper level sets decomposition. This section includes also some examples on the application of such filters. The paper is concluded with a 
summary and perspectives in Section 5 .

2. Background. The aim of this section is to provide the background material necessary for our contribution. More precisely, it is reviewed, on the one hand, the notion of counter-harmonic mean filter and its appropriateness to approximate flat dilation/erosion. On the other hand, the principles of bilateral filtering are also reminded.

2.1. Counter-Harmonic filter. The counter-harmonic mean (CHM) belongs to the family of the power means, which are studied in detail in [12]. The CHM has been considered in the state-of-the-art of image processing as an appropriate filter to deal with salt and pepper noise [22]. For the simplicity of subsequent analysis, let us consider a non-negative valued grey-level image, $f \in \mathcal{F}(E,[0,+\infty])$. In mathematical terms, the CHM filter is defined as follows.

DEFinition 2.1. The CHM filter is obtained as

$$
\kappa_{B}^{P}(f)(\mathbf{x})= \begin{cases}\frac{\sum_{\mathbf{z} \in B(\mathbf{x})} f(\mathbf{z})^{P+1}}{\sum_{\mathbf{z} \in B(\mathbf{x})} f(\mathbf{z})^{P}} & \text { if } P \in \mathbb{R} \\ \max _{\mathbf{z} \in B(\mathbf{x})}(f(\mathbf{z}))=\delta_{B}(f)(\mathbf{x}) & \text { if } P=+\infty \\ \min _{\mathbf{z} \in B(\mathbf{x})}(f(\mathbf{z}))=\varepsilon_{B}(f)(\mathbf{x}) & \text { if } P=-\infty\end{cases}
$$

where $f^{P}(\mathbf{x})$ is the pixel image value $f(\mathbf{x})$ to the power of $P$ and $B(\mathbf{x})$ is the window of the filter centered at point $\mathbf{x}$, i.e., the structuring element in the case of morphological operators.

This filter is well suited for reducing the effect of pepper noise for $P>0$ and of salt noise for $P<0$. It is easy to see that for $P \gg 0(P \ll 0)$ the pixels with largest (smallest) values in the local neighborhood $B$ will dominate the result of the weighted sum. Of course, in practice, the range of $P$ is limited due to the precision in the computation of the floating point operations. In the pioneering paper [53], starting from the natural observation that morphological dilation and erosion are the limit cases of the CHM, it was proposed to use the CHM to calculate robust nonlinear operators which approach the morphological ones but without using max and min operators. In addition, these operators are more robust to outliers (i.e., to noise) and consequently they can be considered as an alternative to rank-based filters in the implementation of pseudo-morphological operators.

In our recent study [2] we have also considered empirically how both means converge to the supremum (resp. infimum) when positive $P$ increases (negative $P$ decreases). Let us examine also two properties which are useful to understand the practical interest of the CHM filter.

Proposition 2.2. If $0 \leq P \leq+\infty$ then $\kappa_{B}^{P}(f) \geq \mathfrak{M}_{B}^{P}(f)$; and if $-\infty \leq P \leq$ 0 then the following stronger results holds: $\kappa_{B}^{P}(f) \leq \mathfrak{M}_{B}^{P-1}(f)$; where $\mathfrak{M}_{B}^{P}(f)=$ $\left(\sum_{\mathbf{z} \in B(\mathbf{x})} f(\mathbf{z})^{P}\right)^{1 / P}$ is the $P$-th power-mean filter, or Minkowski mean of order $P$, defined for $P \in \mathbb{R}^{*}$. Inequalities are strict unless $P=0,+\infty,-\infty$ or if $f(\mathbf{x})$ is constant.

Proposition 2.3. If $-\infty \leq P<Q \leq+\infty$ then $\kappa_{B}^{P}(f) \leq \kappa_{B}^{Q}(f)$, with equality if and only if $f(\mathbf{x})$ is constant.

Proofs of Propositions 2.2 and 2.3 as well as other properties can be found in [12]. Proposition 2.2 justifies theoretically the suitability of $\mathrm{CHM}$ with respect to the alternative approach by high-order Minkowski mean, as considered by Welk [58], in order to propose a nonlinearization of averaging-based filters. We note that according 
to Proposition 2.2, the convergence to the erosion with $P \ll 0$ is faster than to the dilation with equivalent $P \gg 0$, i.e., for $P>0$

$$
\left|\kappa_{B}^{P}(f)(x, y)-\delta_{B}(f)(x, y)\right| \geq\left|\kappa_{B}^{-P}(f)(x, y)-\varepsilon_{B}(f)(x, y)\right|, \quad \forall(x, y) \in \Omega
$$

This asymmetry indicates that $\kappa_{B}^{P}(f)$ and $\kappa_{B}^{-P}(f)$ are not dual operators with respect to the complement, i.e., for $P>0$

$$
\kappa_{B}^{P}(f) \neq \complement \kappa_{B}^{-P}(\complement f)
$$

with $\complement f=1-f$. As it was already pointed out in [53], the fundamental drawback of $\kappa_{B}^{P}(f)$ (resp. $\left.\kappa_{B}^{-P}(f)\right)$ is the fact that $f(\mathbf{x}) \not \leq \kappa_{B}^{P}(f)(\mathbf{x})$ with $P>0$ (resp. $f(\mathbf{x}) \nsupseteq$ $\kappa_{B}^{-P}(f)(\mathbf{x})$ with $\left.P<0\right)$. Or in other words, the extensitivity (resp. anti-extensitivity) for $P>0$ (resp. $P<0$ ) is not guaranteed. However, according to proposition 2.3, the following ordering relationship holds for $P>0$ :

$$
\kappa_{B}^{-P}(f)(x, y) \leq \kappa_{B}^{P}(f)(x, y) .
$$

2.2. Bilateral filter: spatially-variant convolution kernels. Canonic multiscale image analysis involves obtaining the various scales by linear convolutions (i.e., low-pass filtering) of the original image. Hence, each output image pixel value is a weighted sum of its neighbors in the input images, where the weights decrease with the spatial distance to the center position. These weights are usually given by a Gaussian kernel, which is also related to linear diffusion. As a result, all neighborhoods are uniformly blurred, including edges and discontinuities.

The rationale behind the bilateral filtering is to apply a weighted average of nearby pixels which depends on the distance but also on the difference in value with respect to the center.

DEFINITION 2.4. The bilateral filter of image $f(\mathbf{x})$ with spatially-variant bivariate kernel $\widetilde{k}_{\eta_{s}, \eta_{i}}(\xi)$ is defined by the following normalized convolution:

$\mathrm{BL}(f)\left(\mathbf{x} ; \widetilde{k}_{\eta_{s}, \eta_{i}}\right)=\frac{\int_{E} f(\mathbf{y}) \widetilde{k}_{\eta_{s}, \eta_{i}}(\mathbf{x}-\mathbf{y}) d \mathbf{y}}{\int_{E} \widetilde{k}_{\eta_{s}, \eta_{i}}(\mathbf{x}-\mathbf{y}) d \mathbf{y}}=\frac{\int_{E} f(\mathbf{y}) k_{\eta_{s}}(\|\mathbf{x}-\mathbf{y}\|) k_{\eta_{i}}\left(\left|f_{\rho}(\mathbf{x})-f_{\rho}(\mathbf{y})\right|\right) d \mathbf{y}}{\int_{E} k_{\eta_{s}}(\|\mathbf{x}-\mathbf{y}\|) k_{\eta_{i}}\left(\left|f_{\rho}(\mathbf{x})-f_{\rho}(\mathbf{y})\right|\right) d \mathbf{y}}$

where the pair of width parameters defines the vector scale $\left(\eta_{s}, \eta_{i}\right)$, with $\eta_{s}$ is the spatial (or size) scale and $\eta_{i}$ the intensity (or tonal or range) scale; and where $k_{\eta}(\xi)$ denotes the function kernel which imposes the smoothing weights of both spatial and contrast effects.

Thus, in the bilateral filter, each neighbor is weighted by a spatial component that penalizes distant pixels and an intensity component that penalizes pixels with a different intensity. The combination by product of both components ensures in $\mathrm{BL}(f)$ that only nearby similar pixels contribute to the final results. In this work, it will be considered for simplicity that the spatial and intensity kernels are the same. The intensity distances are computed from $f_{\rho}(\mathbf{x})$, a median-filtered version of the initial image $f(\mathbf{x})$ using a window of size $\rho \times \rho$ pixels. The computation of the intensity penalization from $f_{\rho}(\mathbf{x})$, a regularized version of the image, leads to robustness against noise estimator. This approach is well known in nonlinear diffusion [15] and in the computation of morphological amoebas (i.e., the "pilot" image [36]). For all the examples given in this paper, we have fixed $\rho=3$ pixels. As in spatially-invariant filtering, increasing the spatial parameter $\eta_{s}$ smoothes large features. As the intensity 

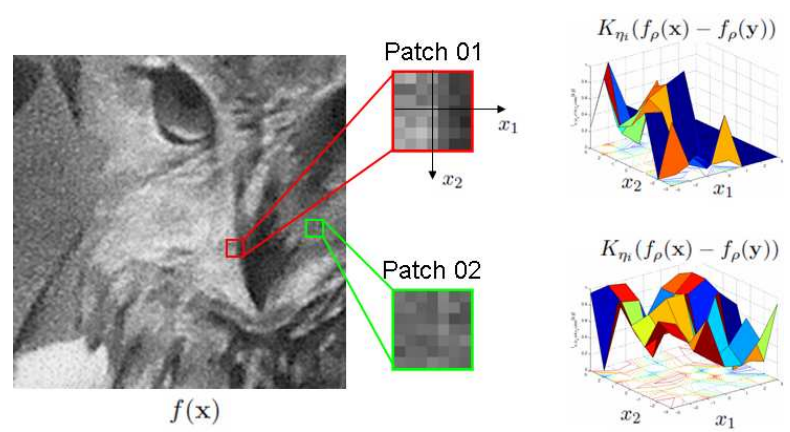

Figure 2.1. Original Owl image $f(\mathbf{x})$ which will be used as illustrative example in the paper. It includes two zoomed-in patches as well as the corresponding local intensity difference terms $k_{\eta_{i}}\left(f_{\rho}(\mathbf{x})-f_{\rho}(\mathbf{y})\right)$, using a Gaussian kernel.

parameter $\eta_{i}$ increases, the bilateral filter gradually approximates spatially-invariant filtering, but if the value is too small, no smoothing occurs.

Fig. 2.1 depicts an image that is used as an illustrative example throughout the paper. Figure includes two zoomed-in patches as well as the corresponding local intensity difference terms $k_{\eta_{i}}\left(f_{\rho}(\mathbf{x})-f_{\rho}(\mathbf{y})\right)$, using a Gaussian kernel. In the standard formulation of bilateral filtering, the kernel is a Gaussian function: it is well known that the Gaussian convolution yields a least squares zero-order estimate of image structure and the Gaussian-based bilateral convolution leads to a robust estimate of local image structure $[18,8]$. However, for the nonlinearization case studied in this paper, we propose to consider in detail three alternative kernels $k_{\sigma}(\xi)$, which correspond in fact to three of the most useful continuous probability distributions [31]:

- Gaussian kernel: $k_{\sigma}(\xi)=e^{\frac{-\xi^{2}}{2 \sigma^{2}}}$

- Laplace kernel: $k_{\beta}(\xi)=e^{\frac{-|\xi|}{\beta}}$

- Cauchy-Lorentz kernel: $k_{\alpha}(\xi)=\frac{1}{1+\frac{\xi^{2}}{\alpha^{2}}}$

Let us just justify the choice of these three kernels. Besides the Gaussian kernel, which involves the optimal weights for denoising Gaussian-like noise according to the MAP criterion, the Laplace kernel can be considered as the $L_{1}$ counterpart and consequently be more robust for the estimation of adaptive kernels in case of impulse noise. Both are exponential kernels, and as we will show later, their "morphologization" implies to compute their logarithm to obtain respectively a parabolic and a conic structuring function. The Cauchy-Lorentz kernel is an intermediate function between the Gaussian and Laplace kernels, it is sharper than the Gaussian kernel; its main property is its heavy-tailed shape distribution. This last point is important in bilateral kernel since an adaptability more focused on the range penalization.

It is easy to see that the three kernels are bounded positive functions, i.e., $0 \leq k_{\eta}(\xi) \leq 1, \xi \in \mathbb{R}$. Note also that as the convolution is normalized in the bilateral filtering, the corresponding kernels do not require any additional normalization. Fig. 2.2 provides an example of the 2D spatial kernel $k_{\eta_{s}}(\|\mathbf{x}-\mathbf{y}\|)$ for the three considered cases, where the width parameter is $\eta_{s}=2$ in the three cases. Then, the corresponding bilateral kernels, locally adaptive, for Patch 01 of "Owl" image (see Fig. 2.1), after weighting the shape function with the intensity distance kernel $k_{\eta_{i}}\left(\left|f_{\rho}(\mathbf{x})-f_{\rho}(\mathbf{y})\right|\right)$, with $\eta_{i}=0.1$. For an easier visual comparison, a 1D section of the spatial and bilateral kernels is also provided. We note that for a convenient inter- 
pretation of the intensity and bilateral kernels, the image values have been normalized to the interval $[0,1]$. As previously pointed out, we observe that the Cauchy-Lorentz kernel is an intermediate function between the Gaussian and Laplace kernels, and consequently it is sharper than the Gaussian kernel.

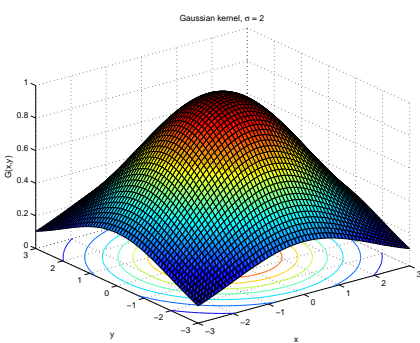

(a)

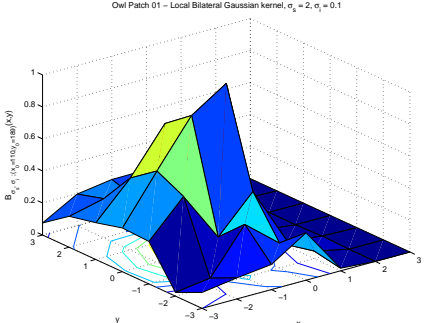

(d)

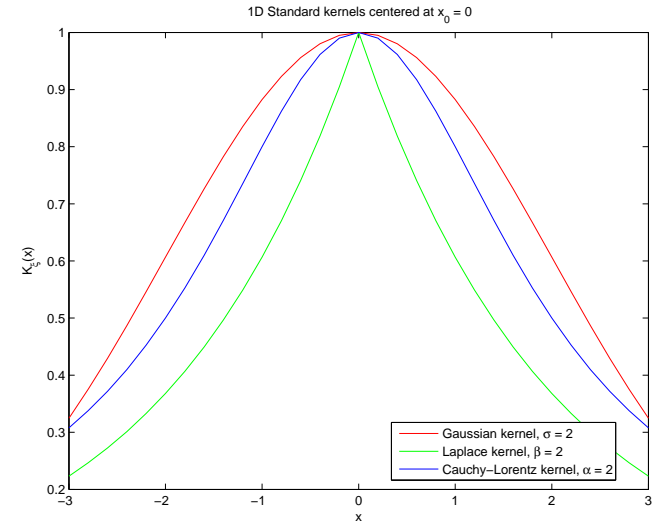

$(\mathrm{g})$

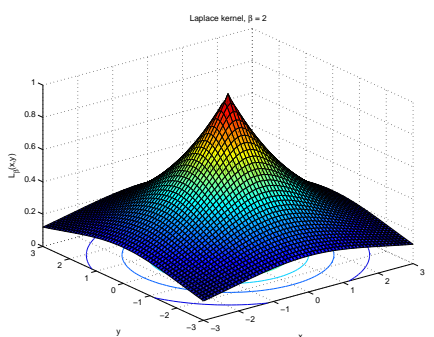

(b)

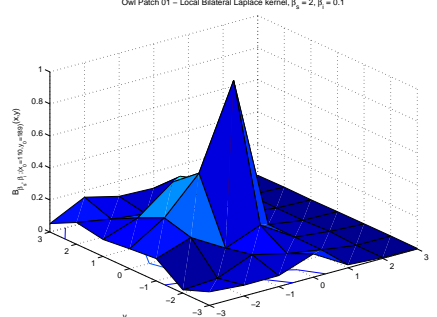

(e)

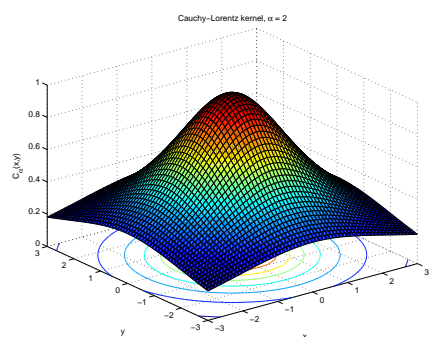

(c)

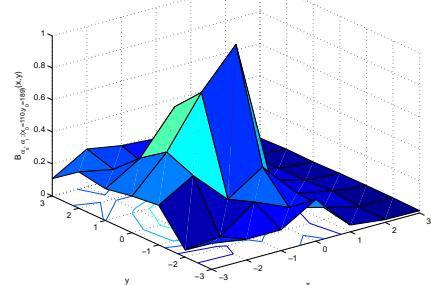

(f)

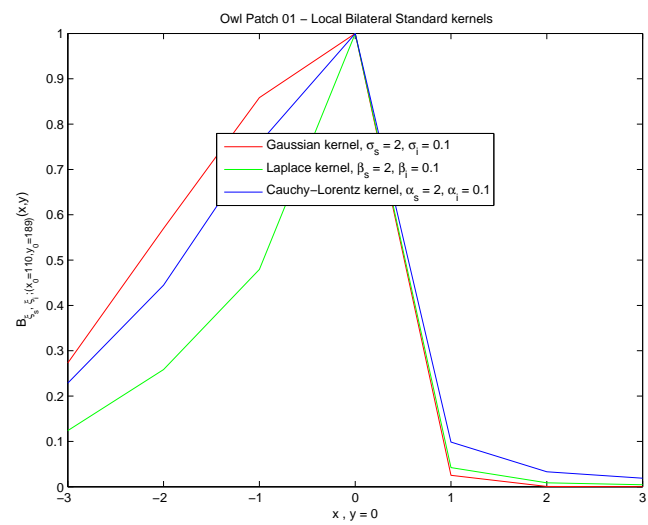

(h)

FiguRE 2.2. First row: 2D Gaussian kernel (a), Laplacian kernel (b) and Cauchy-Lorentz kernel (c). Second row: 2D adaptive bilateral Gaussian kernel (d), Laplacian (e) and CauchyLorentz $(f)$, for Patch 01 from Owl image (see Fig. 2.1). Third row: $1 D$ section of the spatial kernels $(g)$ and of the bilateral kernels for the patch $01(h)$. The scale parameters are $\eta_{s}=2$ and $\eta_{i}=0.1$.

3. Counter-Harmonic Bilateral filter. This section introduces the proposed counter-harmonic generalization of bilateral filtering, including a study on the limit relationships with locally adaptive non-linear morphological operators that behave like morphological filters. We introduce also the notion of bilateral parabolic structuring function. 
3.1. Counter-Harmonic generalization. Utilizing the counter-harmonic paradigm reviewed in previous Section, the following generalized bilateral filter is proposed.

DEFINITION 3.1. The counter-harmonic bilateral filter of order $P$ using bilateral kernel $\widetilde{k}_{\eta_{s}, \eta_{i}}$ is defined as

$$
\Psi(f)\left(\mathbf{x} ; \widetilde{k}_{\eta_{s}, \eta_{i}} ; P\right)=\frac{\int_{E} f(\mathbf{y})^{P+1} k_{\eta_{s}}(\|\mathbf{x}-\mathbf{y}\|) k_{\eta_{i}}\left(\left|f_{\rho}(\mathbf{x})-f_{\rho}(\mathbf{y})\right|\right) d \mathbf{y}}{\int_{E} f(\mathbf{y})^{P} k_{\eta_{s}}(\|\mathbf{x}-\mathbf{y}\|) k_{\eta_{i}}\left(\left|f_{\rho}(\mathbf{x})-f_{\rho}(\mathbf{y})\right|\right) d \mathbf{y}} .
$$

By choosing $P>0$ (resp. $P<0), \Psi(f)\left(\mathbf{x} ; \widetilde{k}_{\eta_{s}, \eta_{i}} ; P\right)$ leads to a parameterized family of max-activity (resp. min-activity) operators, which filtering effects for a given pair of spatial/distance scales $\left(\eta_{s}, \eta_{i}\right)$ depend on the "nonlinearity order" of P. According to the value of $P$, this filter skews the bilateral weighted values towards the supremum or infimum value.

Fig. 3.1 depicts a comparative example of filtering the image "Owl" using the counter-harmonic bilateral filter $\Psi(f)\left(\mathbf{x} ; \widehat{k}_{\eta_{s}, \eta_{i}} ; P\right)$, for a Gaussian kernel and a fixed spatial scale $\sigma_{s}=3$ (windowed in a spatial support of $11 \times 11$ pixels), three intensity scales $\sigma_{i}=0.9,0.1,0.01$ and three values of $P>0$; besides the standard bilateral filtering $(P=0)$ and the flat dilation $(P=+\infty)$ of size $11 \times 11$. The behavior of bilateral filtering with respect to the value of $\sigma_{i}$ is here well illustrated: with high values, e.g., $\sigma_{i}=0.9$, the result is similar to the spatially-invariant Gaussian filter; with low values, e.g., $\sigma_{i}=0.01$, the filtering effect is reduced, independently of the size of the spatial kernel; $\sigma_{i}=0.1$ is here a good trade-off to achieve the adaptive effect of bilateral kernels.

We observe also the effect of adaptive max-activity filter when $P>0$, for instance with $P=5$ : the spatially-variant dilation results in a weighting moving window max. on regular regions, but without distorting the main edges. We note that when $P \uparrow \uparrow$, the bilateral max-activity filter converges to the spatially-invariant flat dilation, losing the properties of local adaptability. In Fig. 3.2 is provided another comparison of max-activity filtering of image $f(\mathbf{x})$ "Owl", for a fixed nonlinear order $P=10$ and an intensity scale $\sigma_{i}=0.1$. In this case, the results for four different spatial scales are depicted; the standard size-equivalent flat dilations are also included. We observe that the adaptivity property in max-activity filter yields stable image results with respect to changes in the spatial scale. It should be remarked that a series of bilateral filters does not produce a scale-space since the standard semigroup law (recursivity principle) associated to Gaussian convolution does not hold, i.e., $\Psi\left(\Psi(f)\left(\mathbf{x} ; \widetilde{k}_{t_{1}, \eta_{i}} ; P\right)\right)\left(\mathbf{x} ; \widetilde{k}_{t_{2}, \eta_{i}} ; P\right) \neq \Psi(f)\left(\mathbf{x} ; \widetilde{k}_{t_{1}+t_{2}, \eta_{i}} ; P\right)$.

Due to the fact that the nonlinear filter $(3.1)$ for $P>0$ (resp. $P<0$ ) is not extensive, i.e., $\Psi(f)\left(\mathbf{x} ; \widetilde{k}_{\eta_{s}, \eta_{i}} ; P>0\right) \nsupseteq f(\mathbf{x}), \forall \mathbf{x} \in E$ (resp. anti-extensive) and does not commute with the supremum, i.e., $\Psi\left(\bigvee_{n}\left(f_{n}\right)\left(\mathbf{x} ; \widetilde{k}_{\eta_{s}, \eta_{i}} ; P\right) \neq \bigvee_{n} \Psi\left(f_{n}\right)\left(\mathbf{x} ; \widetilde{k}_{\eta_{s}, \eta_{i}} ; P\right)\right.$, $\forall \mathbf{x} \in E$ (resp. with the infimum), it cannot be considered stricto sensu as a dilation (resp. erosion). This is the reason why the terminology of max-activity and minactivity filters has been adopted. But let us contemplate in detail the asymptotic behavior of the bilateral $\max /$ min-activity filters with respect to $P$.

3.2. Limit statements and spatially-variant structuring functions. We know from the definition of CHM that

$$
\Psi(f)\left(\mathbf{x} ; \widetilde{k}_{\eta_{s}, \eta_{i}} ; P=+\infty\right)=\sup _{\mathbf{y} \in E}(f(\mathbf{x}-\mathbf{y}))=\delta_{B}(f)(\mathbf{x})
$$




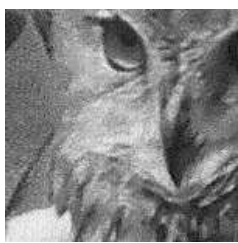

(a) Original

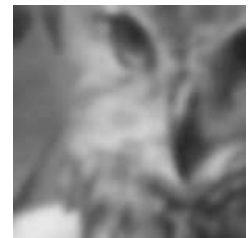

(c1) $\sigma_{s}=3, \sigma_{i}=0.9$ $P=0$

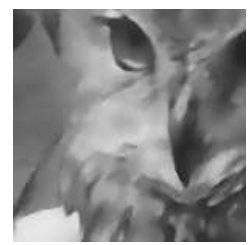

(c1) $\sigma_{s}=3, \sigma_{i}=0.1$ $P=0$

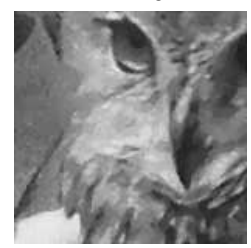

(c1) $\sigma_{s}=3, \sigma_{i}=0.01$ $P=0$

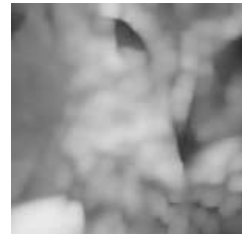

(c2) $\sigma_{s}=3, \sigma_{i}=0.9$ $P=5$

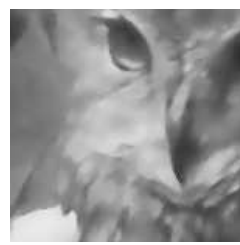

(c2) $\sigma_{s}=3, \sigma_{i}=0.1$ $P=5$

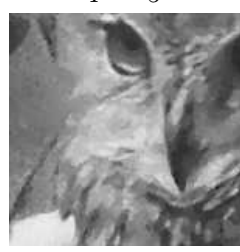

(c2) $\sigma_{s}=3, \sigma_{i}=0.01$ $P=5$

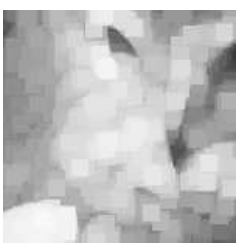

(b) Dilation: $P=+\infty$

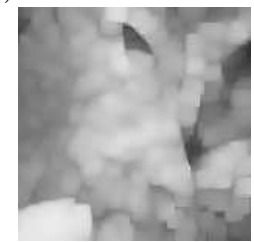

(c3) $\sigma_{s}=3, \sigma_{i}=0.9$ $P=10$

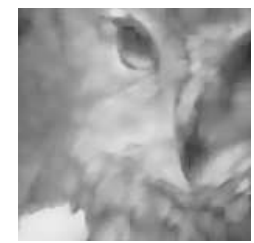

(c3) $\sigma_{s}=3, \sigma_{i}=0.1$ $P=10$

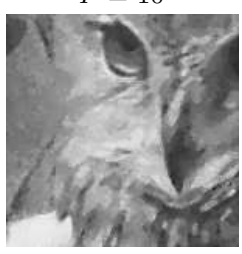

(c3) $\sigma_{s}=3, \sigma_{i}=0.01$ $P=10$

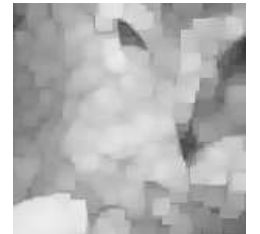

(c4) $\sigma_{s}=3, \sigma_{i}=0.9$ $P=20$

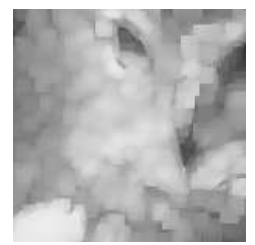

(c4) $\sigma_{s}=3, \sigma_{i}=0.1$ $P=20$

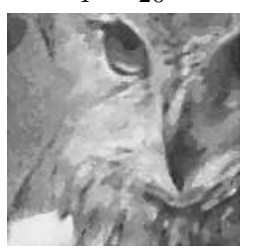

(c4) $\sigma_{s}=3, \sigma_{i}=0.01$ $P=20$

FIGURE 3.1. Comparison of max-activity filtering of image $f(\mathbf{x})$ "Owl" using the counterharmonic bilateral filter $\Psi(f)\left(\mathbf{x} ; \widetilde{k}_{\eta_{s}, \eta_{i}} ; P\right)$, for a Gaussian kernel and a fixed spatial scale $\sigma_{s}=3$ (windowed in a spatial support of $11 \times 11$ pixels), three intensity scales $\sigma_{i}=0.9,0.1,0.01$ and three values of $P>0$; besides the standard bilateral filtering $(P=0)$ and the flat dilation $(P=+\infty)$ of size $11 \times 11$.

and

$$
\Psi(f)\left(\mathbf{x} ; \widetilde{k}_{\eta_{s}, \eta_{i}} ; P=-\infty\right)=\inf _{\mathbf{y} \in E}(f(\mathbf{x}-\mathbf{y}))=\varepsilon_{B}(f)(\mathbf{x}),
$$

i.e., flat dilation and flat erosion, where $B$ is the spatial square support of the bilateral kernel. We can study also the limit cases for $P \gg 0$ and $P \ll 0$.

Proposition 3.2. For a given pair of scale parameters $\left(\eta_{s}, \eta_{i}\right)$, the first order approximation of operator $\Psi(f)\left(\mathbf{x} ; \widetilde{k}_{\eta_{s}, \eta_{i}} ; P\right)$ with respect to $P$, before the limit cases have following behavior:

$$
\begin{aligned}
& \Psi(f)\left(\mathbf{x} ; \widetilde{k}_{\eta_{s}, \eta_{i}} ; P>>0\right) \approx \\
& \quad \sup _{\mathbf{y} \in E}\left\{f(\mathbf{y})+\frac{1}{P}\left[\log \left(k_{\eta_{s}}(\|\mathbf{x}-\mathbf{y}\|)\right)+\log \left(k_{\eta_{i}}\left(\left|f_{\rho}(\mathbf{x})-f_{\rho}(\mathbf{y})\right|\right)\right)\right]\right\}
\end{aligned}
$$




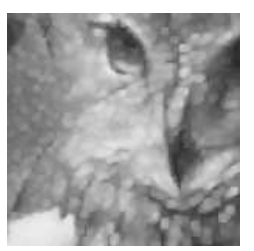

(a1) $\sigma_{s}=1.5, \sigma_{i}=0.1$ $P=10$

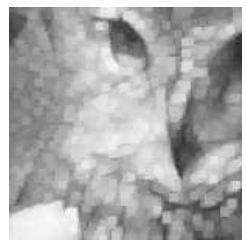

(b1) $\sigma_{s}=1.5, \sigma_{i}=0.1$ $P=+\infty$

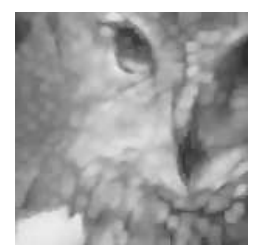

(a2) $\sigma_{s}=2, \sigma_{i}=0.1$ $P=10$

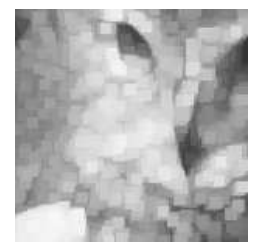

(b2) $\sigma_{s}=2, \sigma_{i}=0.1$

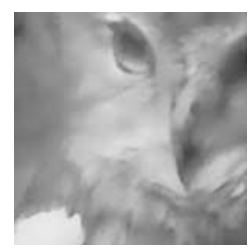

(a3) $\sigma_{s}=7, \sigma_{i}=0.1$ $P=10$

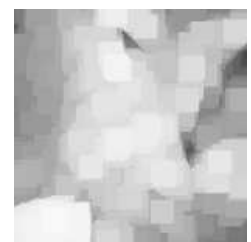

(b3) $\sigma_{s}=7, \sigma_{i}=0.1$

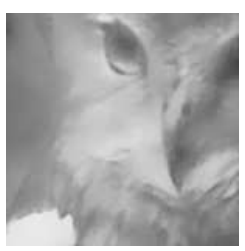

(a4) $\sigma_{s}=10, \sigma_{i}=0.1$ $P=10$

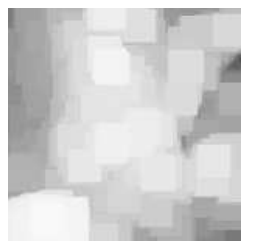

(b4) $\sigma_{s}=10, \sigma_{i}=0.1$

FIGURE 3.2. Comparison of max-activity filtering of image $f(\mathbf{x})$ "Owl" using the counterharmonic bilateral filter $\Psi(f)\left(\mathbf{x} ; \widetilde{k}_{\eta_{s}, \eta_{i}} ; P\right)$ with $P=10$, for a Gaussian kernel and a fixed intensity scale $\sigma_{i}=0.1$ and four spatial scales $\sigma_{s}=1.5$ (windowed in a spatial support of $5 \times 5$ pixels), $\sigma_{s}=2$ $(7 \times 7$ pixels $), \sigma_{s}=5.5(15 \times 15$ pixels $)$ and $\sigma_{s}=2(21 \times 21$ pixels $)$. The corresponding flat dilations $(P=+\infty)$ are also included.

$$
\begin{aligned}
& \Psi(f)\left(\mathbf{x} ; \widetilde{k}_{\eta_{s}, \eta_{i}} ; P<<0\right) \approx \\
& \quad \inf _{\mathbf{y} \in E}\left\{f(\mathbf{y})-\frac{1}{P}\left[\log \left(k_{\eta_{s}}(\|\mathbf{x}-\mathbf{y}\|)\right)+\log \left(k_{\eta_{i}}\left(\left|f_{\rho}(\mathbf{x})-f_{\rho}(\mathbf{y})\right|\right)\right)\right]\right\}
\end{aligned}
$$

which can be interpreted respectively as the supremal convolution (epigraphic addition) and infimal convolution (notions from convex analysis [44, 27]) of $f(\mathbf{x})$ with a spatially-variant structuring function:

$$
\widetilde{b}\left(\mathbf{x} ; \eta_{s}, \eta_{i} ; P\right)=\frac{1}{P}\left[\log \left(k_{\eta_{s}}(\|\mathbf{x}-\mathbf{y}\|)\right)+\log \left(k_{\eta_{i}}\left(\left|f_{\rho}(\mathbf{x})-f_{\rho}(\mathbf{y})\right|\right)\right)\right]=\frac{1}{P} b_{\eta_{s}, \eta_{i}}(\mathbf{x}),
$$

where

$$
b_{\eta_{s}, \eta_{i}}(\mathbf{x})=\left[b_{\eta_{s}}(\|\mathbf{x}-\mathbf{y}\|)+b_{\eta_{i}}\left(\left|f_{\rho}(\mathbf{x})-f_{\rho}(\mathbf{y})\right|\right)\right] .
$$

Proof. We give only the proof for the case $P \rightarrow+\infty$ since the case $P \rightarrow-\infty$ is exactly similar. By rewriting $f^{P}=\exp (P \log (f))$, taking first order Taylor expansion $\log (f) \approx f-1$ and first order Taylor expansion of exponential function such that

$$
\frac{N}{D}=\exp \left(\log \left(\frac{N}{D}\right)\right) \approx 1+\log (N)-\log (D)
$$

we have:

$$
\begin{gathered}
\lim _{P \rightarrow+\infty} \Psi(f)\left(\mathbf{x} ; \widetilde{k}_{\eta_{s}, \eta_{i}} ; P\right)= \\
1+\log \int_{E} \exp \left((P+1)\left[f(\mathbf{y})+\frac{1}{P+1} \log \left(k_{\eta_{s}}(\|\mathbf{x}-\mathbf{y}\|)\right)+\frac{1}{P+1} \log \left(k_{\eta_{i}}\left(\left|f_{\rho}(\mathbf{x})-f_{\rho}(\mathbf{y})\right|\right)\right)-1\right]\right) d \mathbf{y} \\
+\log \int_{E} \exp \left(P\left[f(\mathbf{y})+\frac{1}{P} \log \left(k_{\eta_{s}}(\|\mathbf{x}-\mathbf{y}\|)\right)+\frac{1}{P} \log \left(k_{\eta_{i}}\left(\left|f_{\rho}(\mathbf{x})-f_{\rho}(\mathbf{y})\right|\right)\right)-1\right]\right) d \mathbf{y},
\end{gathered}
$$




\begin{tabular}{|c|c|c|c|c|}
\hline & $k_{\eta}(\xi)$ & $b_{\eta}(\xi)$ & $\eta_{b}(\xi)$ & $d_{\eta}(\tau)$ \\
\hline Gaussian & $e^{-\frac{\xi^{2}}{2 \sigma^{2}}}$ & $-\frac{\xi^{2}}{2 \sigma^{2}}$ & $\sqrt{-\frac{\xi^{2}}{2 b_{\sigma}}}$ & $(2 \sqrt{-2 \tau}) \sigma$ \\
\hline Laplacian & $e^{-\frac{|\xi|}{\beta}}$ & $-\frac{|\xi|}{\beta}$ & $-\frac{|\xi|}{b_{\beta}}$ & $(-2 \tau) \beta$ \\
\hline Cauchy-Lorentz & $\frac{1}{1+\frac{\xi^{2}}{\alpha^{2}}}$ & $-\log \left(1+\frac{\xi^{2}}{\alpha^{2}}\right)$ & $\sqrt{\frac{\xi^{2}}{e^{-b_{\alpha}}-1}}$ & $\left(2 \sqrt{e^{-\tau}-1}\right) \alpha$ \\
\hline
\end{tabular}

Standard kernel functions $k_{\eta}(\xi)$, associated morphological counterparts $b_{\eta}(\xi)$ and derived parameters: kernel width $\eta$ as function of the morphological kernel value and diameter of the disk $d$ obtained by thresholding the morphological kernel function at value $\tau$, with $\tau \leq 0$.

which can be rewritten as

$$
\begin{gathered}
1+(P+1) \log \left(\int_{E}\left(\exp \left(f(\mathbf{y})+\frac{1}{P+1} \log \left(k_{\eta_{s}}(\|\mathbf{x}-\mathbf{y}\|)\right)+\frac{1}{P+1} \log \left(k_{\eta_{i}}\left(\left|f_{\rho}(\mathbf{x})-f_{\rho}(\mathbf{y})\right|\right)\right)-1\right)\right)^{(P+1)} d \mathbf{y}\right)^{\frac{1}{(P+1)}} \\
+P \log \left(\int_{E}\left(\exp \left(f(\mathbf{y})+\frac{1}{P} \log \left(k_{\eta_{s}}(\|\mathbf{x}-\mathbf{y}\|)\right)+\frac{1}{P} \log \left(k_{\eta_{i}}\left(\left|f_{\rho}(\mathbf{x})-f_{\rho}(\mathbf{y})\right|\right)\right)-1\right)\right)^{P} d \mathbf{y}\right)^{\frac{1}{P}}
\end{gathered}
$$

Using now the standard result

$$
\lim _{P \rightarrow+\infty}\left\{\int_{E} g^{P}(x) d x\right\}^{1 / P}=\sup _{x \in E} g(x),
$$

which holds for positive and bounded function $g$ with support space $E$, and considering continuity and monotonicity of the logarithm, we obtain:

$$
\begin{gathered}
\lim _{P \rightarrow+\infty} \Psi(f)\left(\mathbf{x} ; \widetilde{k}_{\eta_{s}, \eta_{i}} ; P\right)= \\
1+(P+1) \sup _{\mathbf{y} \in E}\left(f(\mathbf{y})+\frac{1}{P+1} \log \left(k_{\eta_{s}}(\|\mathbf{x}-\mathbf{y}\|)\right)+\frac{1}{P+1} \log \left(k_{\eta_{i}}\left(\left|f_{\rho}(\mathbf{x})-f_{\rho}(\mathbf{y})\right|\right)\right)-1\right) \\
+P \sup _{\mathbf{y} \in E}\left(f(\mathbf{y})+\frac{1}{P} \log \left(k_{\eta_{s}}(\|\mathbf{x}-\mathbf{y}\|)\right)+\frac{1}{P} \log \left(k_{\eta_{i}}\left(\left|f_{\rho}(\mathbf{x})-f_{\rho}(\mathbf{y})\right|\right)\right)-1\right) .
\end{gathered}
$$

By considering that both supremum operations gives closer values, and assuming that we have $P>>0$ but that the limit is not reached, we finally obtain the corresponding result. We note that the case $P=+\infty$, which corresponds to the flat dilation, is naturally obtained from the the last expression.

First of all, we observe that the supremal convolution of Rel. (3.4) has exactly the same formulation that the second form of the translation-invariant dilation $\delta_{b}(f)(\mathbf{x})$ as defined in (1.1). We note also that in the case of the erosion, the structuring function should been transposed $b(\mathbf{x})=\widetilde{b}^{*}\left(\mathbf{x} ; \eta_{s}, \eta_{i} ; P\right)$, i.e., $\widetilde{b}^{*}(\mathbf{x} ; \cdot ; \cdot)=\widetilde{b}(-\mathbf{x} ; \cdot ; \cdot)$.

This logarithmic connection, which appears here between the linear and the morphological system theory, has been previously considered in the literature [13], by studying the relationship between the Legendre transform, the Cramer transform and the logarithmic Laplace transform. We note also that, in these limiting cases, the counter-harmonic bilateral framework involves a "normalization" by $P$ of the original spatial and intensity kernel scale parameters during unlinearization, i.e., taking for instance the case of the bilateral Gaussian kernel, the nonlinear asymptotic scale parameters are $\widehat{\sigma_{s}}=\sqrt{P} \sigma_{s}$ and $\widehat{\sigma_{i}}=\sqrt{P} \sigma_{i}$. This result is perfectly coherent with those obtained from totally different paradigms $[58,20]$. Empirically, we have observed that a typical order of magnitude of $5 \leq|P|<10$ yields this limit behavior. We note again that for $P=+\infty \Rightarrow \widehat{\sigma_{s}}=+\infty$, i.e., the structuring function becomes flat, and hence we obtain the flat dilation in a windowed spatial support. 
The corresponding logarithmic kernels $b_{\eta}(\xi)$ for the Gaussian, Laplacian and Cauchy-Lorentz are summarized in Table 3.1, which are respectively a parabolic function, a conic function and a logarithmic Cauchy-Lorentz function. The three kernels, which we will call "morphological kernels", are upper bounded negative functions, i.e., $b_{\eta}(\xi) \leq 0, \xi \in \mathbb{R}$, which are also convex functions. Fig. 3.3 provides a comparison of the three families of kernels, spatial and bilateral for a particular image patch, using the same scale parameters $\eta_{s}=2$ and $\eta_{i}=0.1$.

As we have already stated, the "normalization" introduced by $P$ in the counterharmonic bilateral filtering involves a flattening of the structuring function $\widetilde{b}\left(\mathbf{x} ; \eta_{s}, \eta_{i} ; P\right)$ when $|P|$ increases and consequently, the values of max-activity and min-activity filter for $P \gg 0$ and $P \ll 0$ tends respectively asymptotically to a flat dilation and a flat erosion, which lose the properties of adaptavility. The examples provided in Fig. 3.4 allow one to visualize the asymptotic behaviour of morphological structuring functions with respect to $P$ : we observe that for $P>5$ the kernels are already quite flat, independently of the contribution of the intensity distance-based term. This effect can be observed in the comparison of min-activity filtering of Fig. 3.5, where the results for $P=10$ are roughly similar to the flat erosion. From the comparison of the three kernels at $P=5$, we observe that, as expected, for the same values of spatial and intensity scales, the Log. Cauchy-Lorentz produces intermediate effects between the parabolic and conic structuring functions.

3.3. Bilateral parabolic structuring function. The classical case of the parabolic structuring function as the morphological equivalent of the Gaussian kernel is particularly known in the state-of-the art of mathematical morphology. On the one hand, the parabolic (or more generally, quadratic) structuring functions contain the unique rotationally symmetric structuring functions that can be dimensionally decomposed with respect to the dilation [7] and they lead to the canonic morphological scale-spaces $[28,29]$. On the other hand, parabolic structuring functions are the eigenfunctions, which preserve their shape, of the slope transform $[17,37]$. Slope transform is the equivalent of Fourier transform in the framework of mathematical morphology. However, to our knowledge, this is the first time that the bilateral parabolic structuring function, i.e.,

$$
b_{\sigma_{s}, \sigma_{i}}(\mathbf{x})=-\frac{\|\mathbf{x}-\mathbf{y}\|^{2}}{2 \sigma_{s}^{2}}-\frac{\left|f_{\rho}(\mathbf{x})-f_{\rho}(\mathbf{y})\right|^{2}}{2 \sigma_{i}^{2}}
$$

appears in the literature.

3.4. Towards non-local parabolic structuring functions. The previous counter-harmonic framework can be easily extended to other convolution-based filtering techniques, such as NL-Means. We remind that whereas bilateral filtering builds an estimate of the noise-free image value at a given pixel $\mathbf{x}$ by averaging image values of pixels $\mathbf{y}$ that are located spatially near $\mathbf{x}$ and having similar intensities to $f(\mathbf{x})$, the NL-means algorithm uses pixels $\mathbf{y}$, which can be far in the image from $\mathbf{x}$, but that are "structurally similar" to $\mathbf{x}$. This similarity involves a distance between images patches $\mathcal{N}_{f}(\mathbf{x})$ and $\mathcal{N}_{f}(\mathbf{x})$, where $\mathcal{N}$ is a fixed patch shape of size $N \times N$ pixels centered at the indexed pixel. More precisely, NL-means discrete filter of image $f(\mathbf{x})$ is defined as

$$
N L(f)(\mathbf{x} ; N, \sigma)=\frac{\sum_{\mathbf{y} \in E} \omega_{N, \sigma}(\mathbf{x} ; \mathbf{y}) f(\mathbf{y})}{\sum_{\mathbf{y} \in E} \omega_{N, \sigma}(\mathbf{x} ; \mathbf{y})}
$$




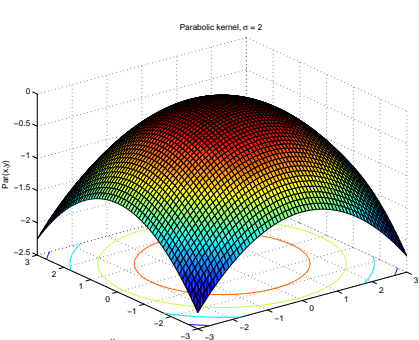

(a)

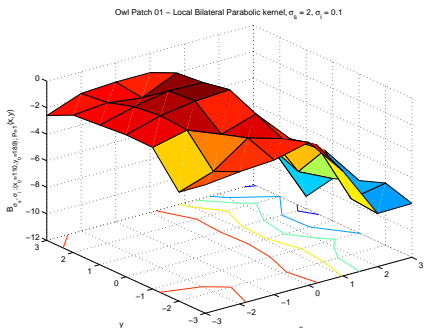

(d)

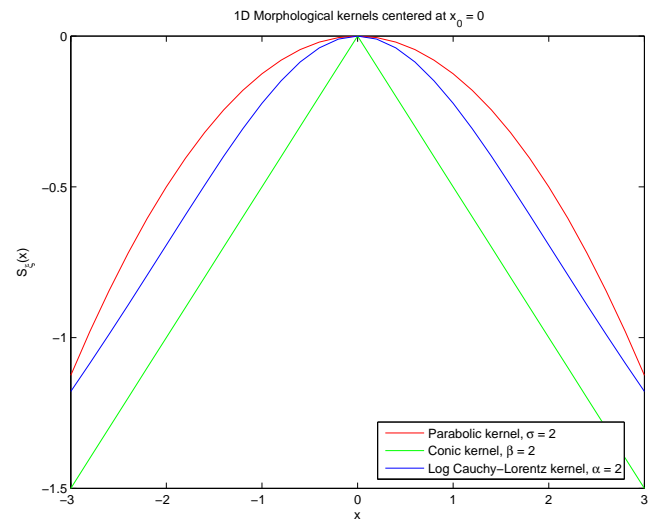

(g)

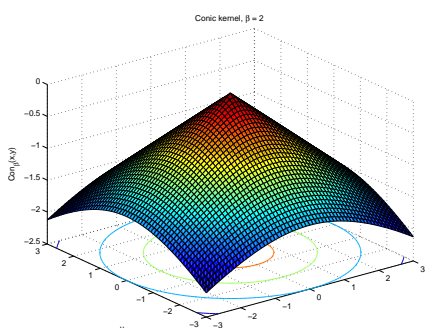

(b)

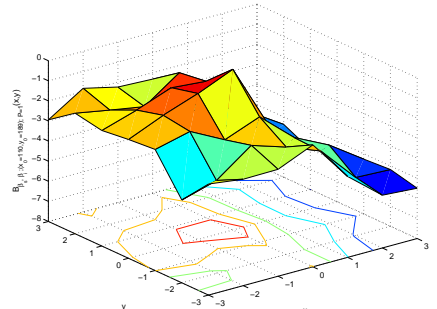

(e)

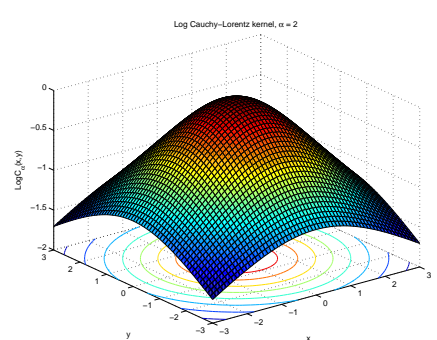

(c)

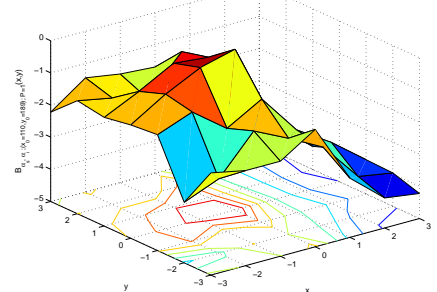

(f)

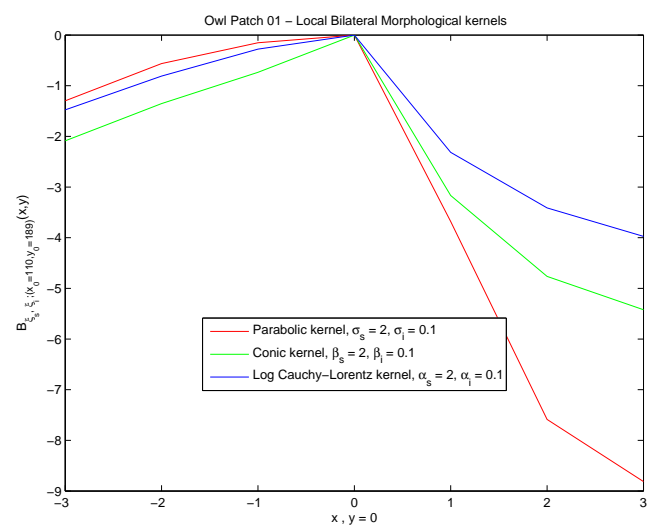

(h)

FiguRE 3.3. First row: 2D parabolic kernel (a), conic kernel (b) and Log. Cauchy-Lorentz kernel (c). Second row: 2D adaptive bilateral parabolic kernel (d), conic (e) and Log. CauchyLorentz $(f)$, for the patch 01 of Owl image (see Fig. 2.1). Third row: 1D section of the spatial morphological kernels $(g)$ and of the bilateral morphological kernels for the patch 01 ( $h$ ). The scale parameters are $\eta_{s}=2$ and $\eta_{i}=0.1$.

where each weight value $\omega_{N, \sigma}(\mathbf{x} ; \mathbf{y})$ is given by the Gaussian kernelized Euclidean distance between the patches:

$$
\omega_{N, \sigma}(\mathbf{x} ; \mathbf{y})=\exp \left(-\frac{d\left(\mathcal{N}_{f}(\mathbf{x}), \mathcal{N}_{f}(\mathbf{y})\right)^{2}}{2 \sigma^{2}}\right) ;
$$

with

$$
d\left(\mathcal{N}_{f}(\mathbf{x}), \mathcal{N}_{f}(\mathbf{y})\right)=\sqrt{\sum_{\mathbf{h} \in \mathcal{N}}(f(\mathbf{x}+\mathbf{h})-f(\mathbf{y}+\mathbf{h}))^{2}} .
$$




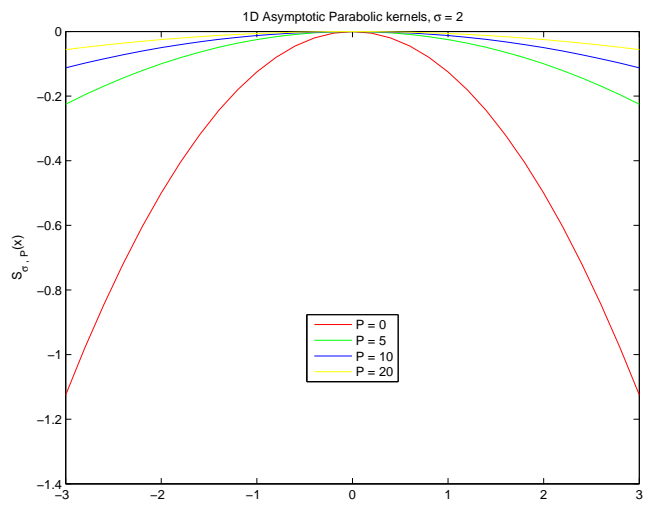

(a)

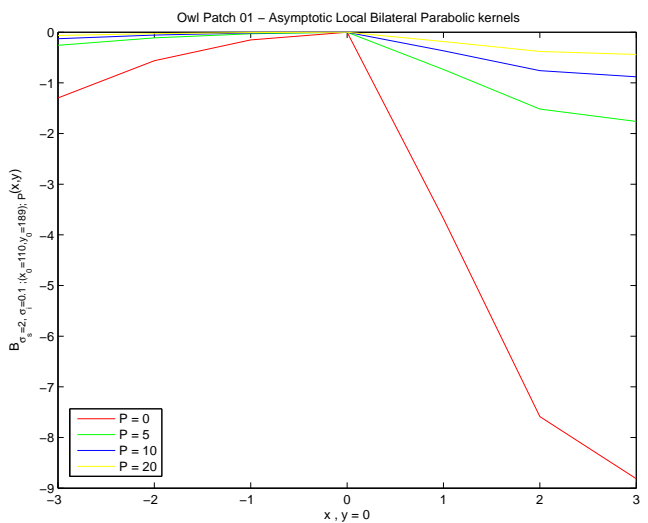

(c)

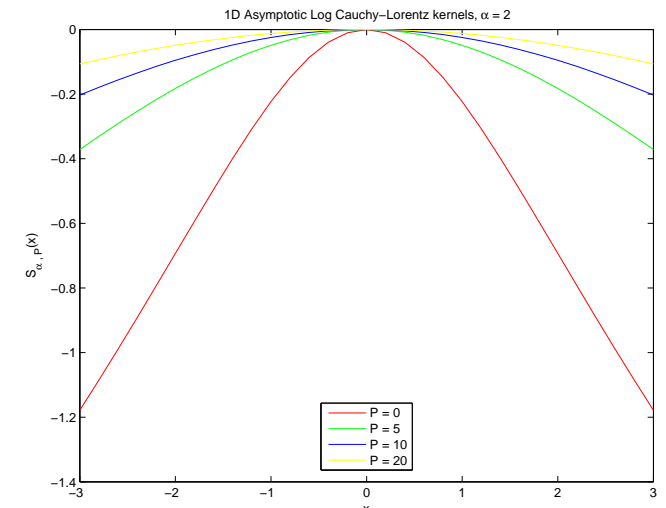

(b)

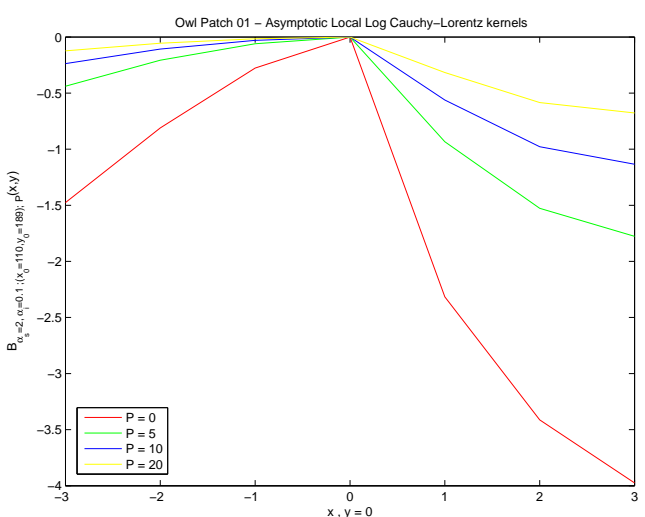

(d)

FIGURE 3.4. Asymptotic behaviour of morphological structuring functions with respect to $P$ : (a) $1 D$ section of spatial parabolic kernel, (b) $1 D$ section of spatial Log. Cauchy-Lorentz kernel, (c) $1 D$ section of adaptive bilateral parabolic kernel for the patch 01 of Owl image, (d) $1 D$ section of adaptive Log. Cauchy-Lorentz parabolic kernel for the patch 01 of Owl image. The scale parameters are $\eta_{s}=2$ and $\eta_{i}=0.1$.

The corresponding counter-harmonic non-local filter of order $P$ is defined in a similar way as (3.1), namely its expression is given by

$$
\Psi^{N L}(f ; N, \sigma ; P)=\frac{\sum_{\mathbf{y} \in E} \omega_{N, \sigma}(\mathbf{x} ; \mathbf{y}) f(\mathbf{y})^{P+1}}{\sum_{\mathbf{y} \in E} \omega_{N, \sigma}(\mathbf{x} ; \mathbf{y}) f(\mathbf{y})^{P}} .
$$

Fig. 3.6 gives an example of non-local max-activity filtering of image "Owl" using the filter $\Psi^{N L}(f ; N, \sigma ; P)$ with three values of $P>0, \sigma=0.1$ and a patch $\mathcal{N}$ of $11 \times 11$ pixels. Using the non-local approach we observe that, in comparison with the bilateral counterpart (see Fig. 3.1 for $\sigma_{i}=0.1$ ), the areas of the image which are similar (e.g., bright zones around the eyes) are dilated similarly. Finally, we can also naturally introduce the non-local parabolic structuring function as

$$
b_{N, \sigma}^{N L}(\mathbf{x})=-\frac{d\left(\mathcal{N}_{f}(\mathbf{x}), \mathcal{N}_{f}(\mathbf{y})\right)^{2}}{2 \sigma^{2}}=-\frac{\sum_{\mathbf{h} \in \mathcal{N}}(f(\mathbf{x}+\mathbf{h})-f(\mathbf{y}+\mathbf{h}))^{2}}{2 \sigma^{2}} .
$$




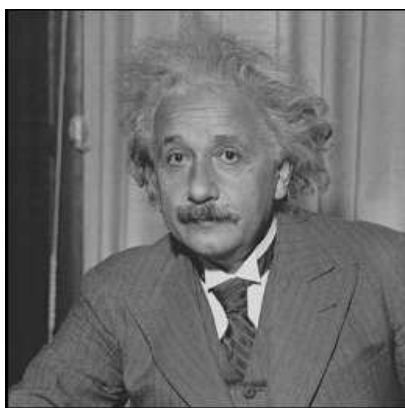

(a) Original

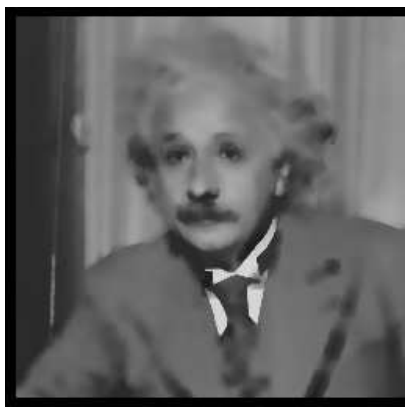

(d1) $\sigma_{s}=3, \sigma_{i}=0.1$ $P=-5$

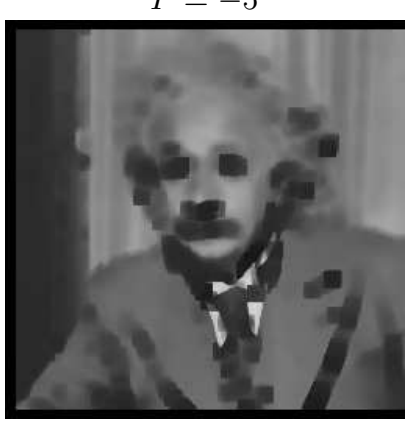

(e1) $\sigma_{s}=3, \sigma_{i}=0.1$ $P=-10$

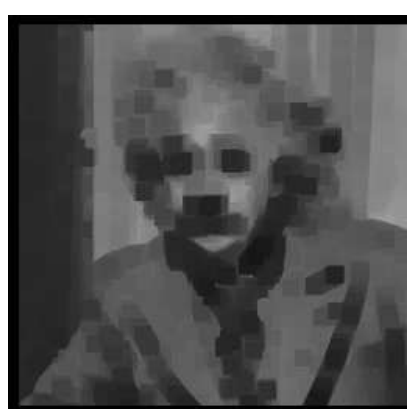

(b) Erosion: $P=-\infty$

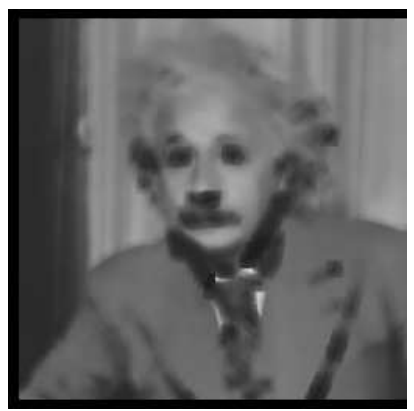

(d2) $\beta_{s}=3, \beta_{i}=0.1$ $P=-5$

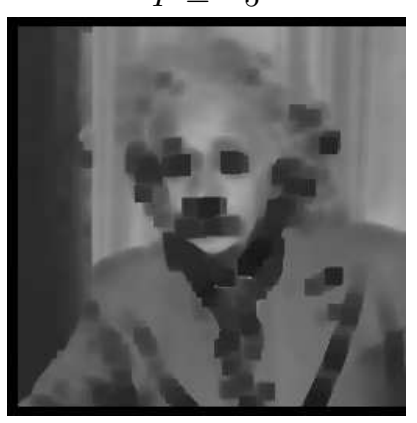

(e2) $\beta_{s}=3, \beta_{i}=0.1$ $P=-10$

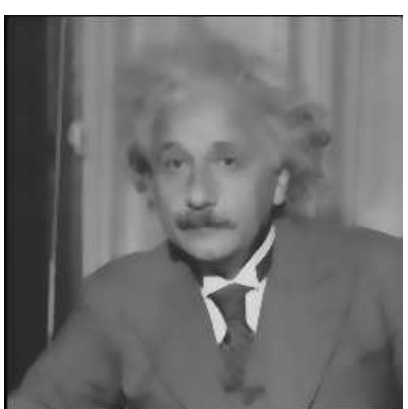

(c) $\sigma_{s}=3, \sigma_{i}=0.1$ $P=0$

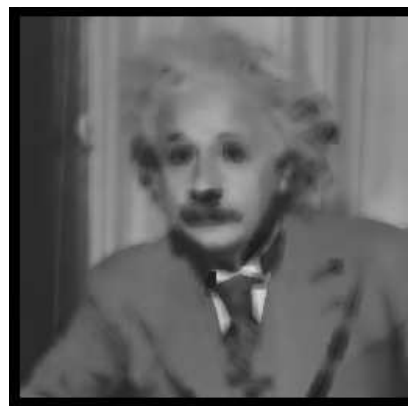

(d3) $\alpha_{s}=3, \alpha_{i}=0.1$ $P=-5$

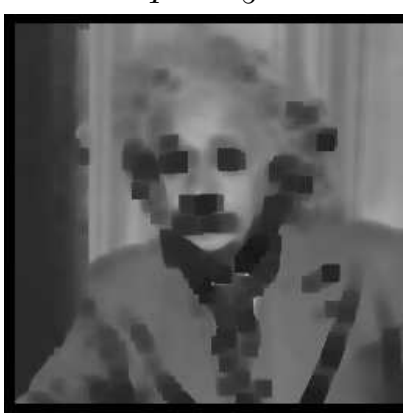

(e3) $\alpha_{s}=3, \alpha_{i}=0.1$ $P=-10$

FIGURE 3.5. Comparison of min-activity filtering of image $f(\mathbf{x})$ "Einstein" using the counterharmonic bilateral filter $\Psi(f)\left(\mathbf{x} ; \widetilde{k}_{\eta_{s}, \eta_{i}} ; P\right)$, using the three kernels (parabolic, conic and Log. Cauchy-Lorentz), for a fixed spatial $\eta_{s}=3$ (windowed in a spatial support of $11 \times 11$ pixels) and intensity $\eta_{i}=0.1$ scales; two values of $P<0$; besides the standard bilateral filtering $(P=0)$ and the flat erosion $(P=-\infty)$ of size $11 \times 11$.

Properties and efficient computation of such non-local parabolic structuring functions will be explored in detail in ongoing research. 


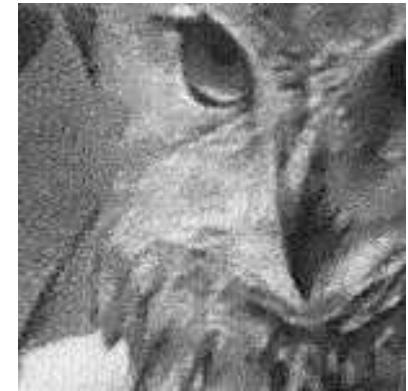

(a) Original

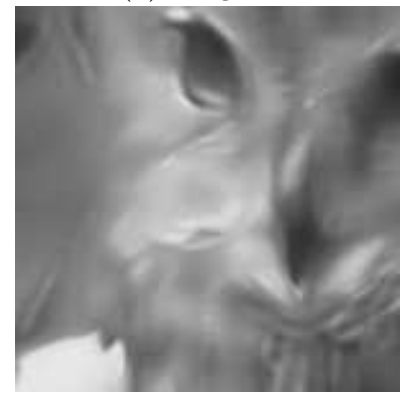

(d) $P=2$

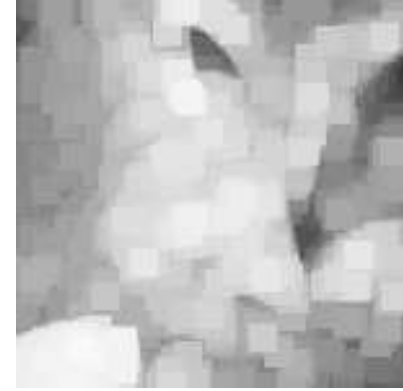

(b) Dilation: $P=+\infty$

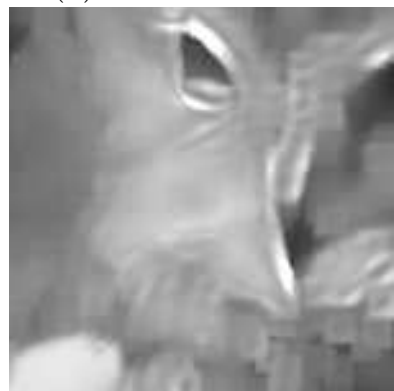

(e) $P=5$

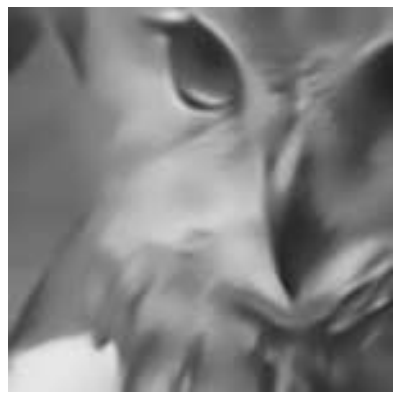

(c) NL-Means: $P=0$

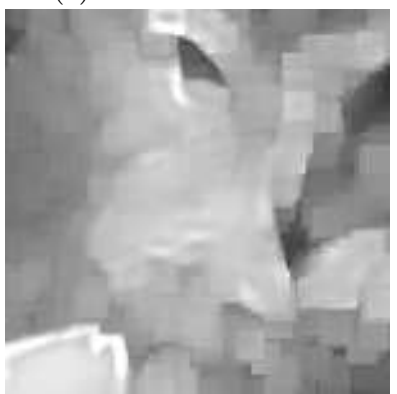

(f) $P=10$

FIGURE 3.6. Comparison of max-activity filtering of image $f(\mathbf{x})$ "Owl", given in (a), using the counter-harmonic non-local filter $\Psi^{N L}(f ; N, \sigma ; P)$, where $\sigma=0.1$ and the patch $\mathcal{N}$ has a size of $11 \times 11$ pixels. Three values of $P>0$ are given in $(d)$ - $(f)$; besides the standard $N L$-means filtering $(P=0)$ and the flat dilation $(P=+\infty)$ of size $11 \times 11$. 
4. Bilateral flat morphological operators. At this point, we have the ingredients to carry out the study on bilateral morphology by considering directly the dilation and erosion using spatially-variant morphological structuring functions [46, 25, 9], without the use of the counter-harmonic framework, i.e.,

$$
\begin{aligned}
& \delta_{b_{\eta_{s}, \eta_{i}}}(f)(\mathbf{x})=\sup _{\mathbf{y} \in E}\left(f(\mathbf{y})+b_{\mathbf{x} ; \eta_{s}, \eta_{i}}(\mathbf{y}-\mathbf{x})\right) \\
& \varepsilon_{b_{\eta_{s}, \eta_{i}}}(f)(\mathbf{x})=\inf _{\mathbf{y} \in E}\left(f(\mathbf{y})-b_{\mathbf{x} ; \eta_{s}, \eta_{i}}(\mathbf{y}+\mathbf{x})\right),
\end{aligned}
$$

where the bilateral structuring function $b_{\mathbf{x} ; \eta_{s}, \eta_{i}}$ is given in Rel.(3.7). The formulation of spatially-variant bilateral opening $\gamma_{b_{\eta_{s}, \eta_{i}}}(f)$ requires the use of the notion of pulse function [26] $i_{\mathbf{x}, t}$ of level $t$ at point $\mathbf{x}$ given by

$$
i_{\mathbf{x}, t}(\mathbf{z})=\left\{\begin{array}{cc}
t & \mathbf{z}=\mathbf{x} \\
-\infty & \mathbf{z} \neq \mathbf{x}
\end{array},\right.
$$

such that any image $g \in \mathcal{F}(E, \mathcal{T})$ can be decomposed into the supremum of its pulses, i.e., $g=\vee\left\{i_{\mathbf{x}, g(\mathbf{x})}, \mathbf{x} \in E\right\}$. Hence, the bilateral opening is then formulated as

$$
\gamma_{b_{\eta_{s}, \eta_{i}}}(f)=\bigvee\left\{C_{(\mathbf{x}, t)}\left(b_{\mathbf{x} ; \eta_{s}, \eta_{i}}\right):(\mathbf{x}, t) \in E \times \mathcal{T}, C_{(\mathbf{x}, t)}\left(b_{\mathbf{x} ; \eta_{s}, \eta_{i}}\right) \leq f\right\}
$$

where the adaptive bilateral cylinder $C_{(\mathbf{x}, t)}\left(b_{\eta_{s}, \eta_{i}}(\mathbf{x})\right)$ the translation of $b_{\eta_{s}, \eta_{i}}(\mathbf{x})$ by $(\mathbf{y}, t)$ is given by

$$
C_{(\mathbf{p}, t)}\left(b_{\mathbf{x} ; \eta_{s}, \eta_{i}}\right)(\mathbf{z})=b_{\mathbf{x} ; \eta_{s}, \eta_{i}}(\mathbf{z}-\mathbf{p})+t
$$

Considering $f$ as a surface in a 3D space, the subgraph of the opening $\gamma_{b_{\eta_{s}, \eta_{i}}}(f)$ is generated by the zone swept by all cylinders $C_{(\mathbf{x}, t)}\left(b_{\mathbf{x} ; \eta_{s}, \eta_{i}}\right)$ smaller than $f$. The bilateral closing $\varphi_{b_{\eta_{s}, \eta_{i}}}(f)$ is defined by duality of the opening.

By its easier geometrical interpretation and its interest for practical applications, the case of flat mathematical morphology deserves a deeper analysis. The purpose of this section is just to introduce the construction of flat spatially-variant morphology using thresholded adaptive structuring functions and a theoretically appropriate formulation of morphological operators.

4.1. Thresholding adaptive structuring function. As we have discussed above, bilateral structuring functions $b_{\eta_{s}, \eta_{i}}(\mathbf{x})$ reflect at each point $\mathbf{x}$ of the image $f(\mathbf{x})$ the neighborhood additive weights for the infimal/supremal convolutions. By thresholding $b_{\eta_{s}, \eta_{i}}$ at a fixed value $\tau$, a neighborhood shape is then obtained for each point $\mathbf{x}$. The binary set $B(\mathbf{x})$, defining an adaptive structuring element, corresponds to a truncated disk. Let us consider in detail a way to define this mapping $f(\mathbf{x}) \mapsto B(\mathbf{x})$ parameterized with geometrical features more meaningful than $\eta_{s}$ and $\eta_{i}$.

Fig. 4.1 shows the shape obtained by thresholding, at three different threshold values $\tau$, the spatial structuring function $b_{\eta_{s}=2}(\mathbf{x})$ and the bilateral structuring function $b_{\eta_{s}=2, \eta_{i}=0.1}(\mathbf{x})$ (the latter for the depicted patch). We observe that, for a given threshold value $\tau$, the size of the spatial shape (i.e., diameter of the disk) is different between the parabolic and Log. Cauchy-Lorentz. In Table 3.1 are given, for the three families of morphological kernels, the expressions for the diameter of the disk $d$ as function of the threshold value and width parameter $\eta_{s}$. Instead of using $\eta_{s}$ as the input parameter to define the spatial scale of the structuring element, we can fix a 


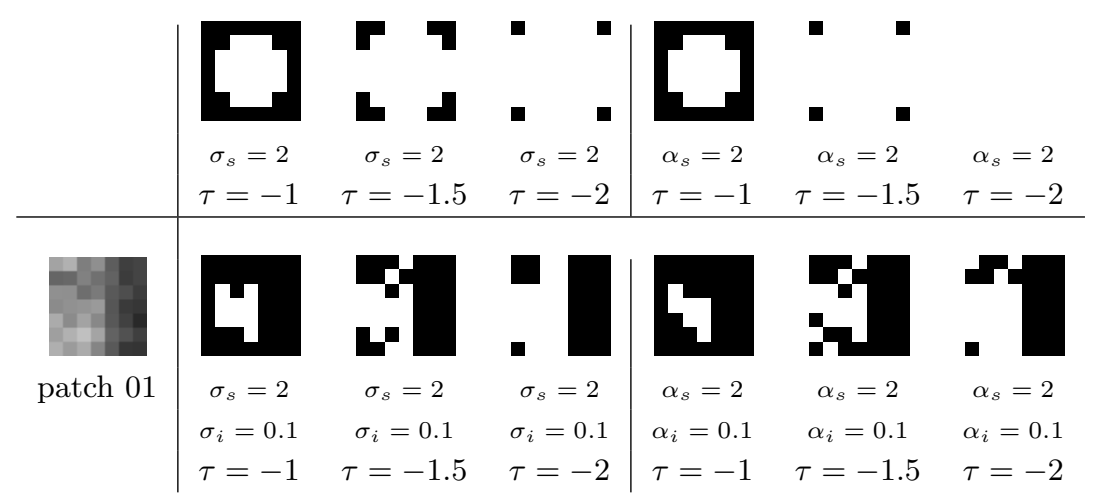

FIGURE 4.1. Upper part, shapes obtained by thresholding, at three different threshold values $\tau$, the spatial structuring function $b_{\eta_{s}=2}(\mathbf{x})$ for two families of kernels: parabolic (middle column) vs. Log. Cauchy-Lorentz (right column). Lower part, given the depicted patch, shape obtained by thresholding, at three different threshold values $\tau$, the bilateral structuring function $b_{\eta_{s}}=2, \eta_{i}=0.1(\mathbf{x})$ for two families of kernels: parabolic (middle column) vs. Log. Cauchy-Lorentz (right column).

constant threshold, for instance $\tau=-1$, and then calculate $\eta_{s}$ for a desired diameter of the disk $d$. Hence, the disk is obtained by $b_{\eta_{s}}(\|\mathbf{x}-\mathbf{z}\|) \geq \tau=-1$, with $\eta_{s}$ computed as function of $d$. But this is only the spatial distance contribution, if we keep the same value for thresholding in the sum of both terms of the bilateral contribution, i.e., $b_{\eta_{s}}(\|\mathbf{x}-\mathbf{z}\|)+b_{\eta_{i}}\left(\left|f_{\rho}(\mathbf{x})-f_{\rho}(\mathbf{z})\right|\right) \geq-1$, the correspond disk will have the nominal diameter for a strictly flat zone, otherwise, some part of the disk will be truncated. To interpret the penalization term, we can use the relation between $\eta_{i}$ and the intensity distance which produces $b_{\eta_{i}}(\mathbf{x})=-1$; that is, the value of $b_{\eta_{i}}(\mathbf{x})$ such that even the closer points to center $\mathbf{x}$ (i.e., $\|\mathbf{x}-\mathbf{z}\| \rightarrow 0$ ) are excluded of the thresholded structuring element. We can introduce indeed a new parameter called critical contrast percentage $\Delta(\%)$. This parameter is the value of $\left|f_{\rho}(\mathbf{x})-f_{\rho}(\mathbf{z})\right|$ producing $b_{\eta_{i}}(\mathbf{x})=-1$ for a certain $\eta_{i}$, which can be therefore calculated.

In summary, the spatially-variant neighborhood shape can be obtained as follows.

DeFinition 4.1. Given an image $f(\mathbf{x})$, the adaptive isotropic structuring element at pixel $\mathbf{x}$ of diameter $d$ and critical contrast percentage $\Delta$ is defined by

$$
B_{d, \Delta}(\mathbf{x})=\left\{\mathbf{z} \in E \mid b_{\eta_{s}}(\|\mathbf{x}-\mathbf{z}\|)+b_{\eta_{i}}\left(\left|f_{\rho}(\mathbf{x})-f_{\rho}(\mathbf{z})\right|\right) \geq-1\right\},
$$

where the corresponding spatial and intensity scale parameters for the morphological Gaussian, Laplace and Cauchy-Lorentz kernels are respectively given by

$$
\sigma_{s}=\frac{d}{2 \sqrt{2}} ; \quad \beta_{s}=\frac{d}{2} ; \quad \alpha_{s}=\frac{d}{2 \sqrt{1.718}} ;
$$

and

$$
\sigma_{i}=\frac{1}{\sqrt{2}} \frac{\Delta}{100} ; \quad \beta_{i}=\frac{\Delta}{100} ; \quad \alpha_{i}=\frac{1}{\sqrt{1.718}} \frac{\Delta}{100}
$$

The adaptive isotropic structuring elements can be straightforwardly applied to calculate for instance a (spatially variant) bilateral flat median filter as follows:

$$
M_{d, \Delta}(f)(\mathbf{x})=\left\{f(\mathbf{y}) \mid f(\mathbf{y})=\operatorname{median}[f(\mathbf{z})], \mathbf{z} \in B_{d, \Delta}(\mathbf{x})\right\}
$$


Fig. 4.2 gives the comparison of bilateral flat median filtering $M_{d, \Delta}(f)(\mathbf{x})$ for image $f(\mathbf{x})$ "Owl" with respect to different values of $d$ and $\Delta$, and using the morphological Gaussian kernel (i.e., parabolic kernel). The quality of the results is comparable to those obtained by the standard bilateral filtering (i.e., adaptive weighting mean) with the advantage that median filter preserves the original set of values. As we show in the example of Fig. 4.5, this assertion is not true for very noisy images, where the standard bilateral filtering outperforms the bilateral flat median filtering.

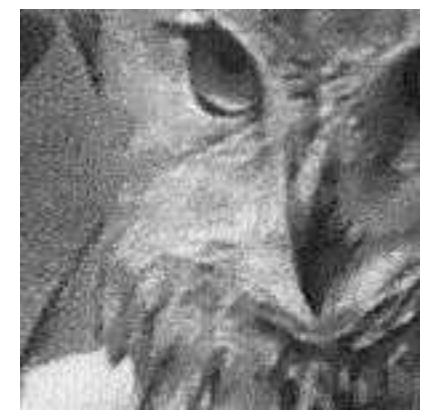

(a) Original

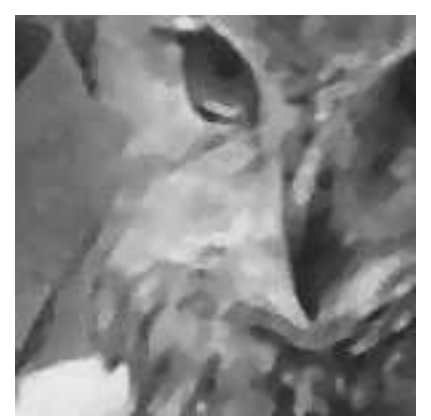

(b) $d=7 ; \Delta=5$

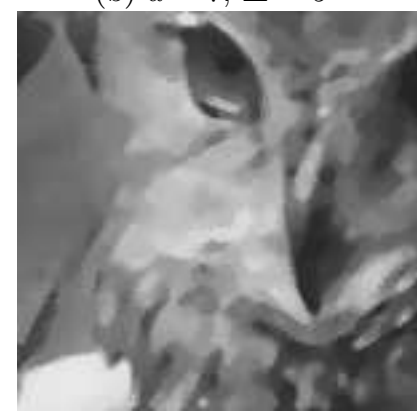

(e) $d=11 ; \Delta=5$

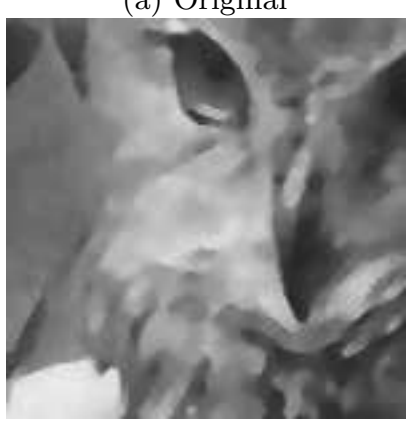

(c) $d=7 ; \Delta=15$

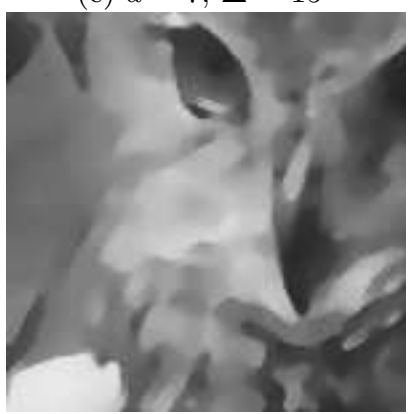

(f) $d=11 ; \Delta=15$

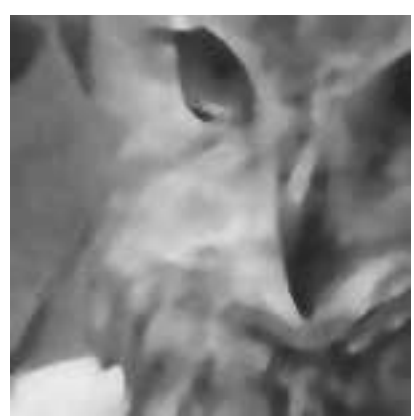

(d) $d=7 ; \Delta=45$

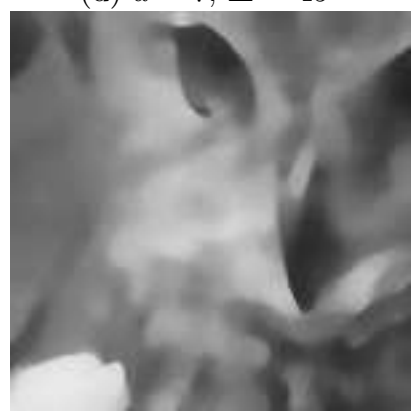

(g) $d=11 ; \Delta=45$

Figure 4.2. Comparison of bilateral flat median filtering $M_{d, \Delta}(f)(\mathbf{x})$ for image $f(\mathbf{x})$ "Owl" (given in (a)) with respect to three different values of $d$ (rows) and three different values of $\Delta$ (columns), and using the morphological Gaussian kernel (i.e., parabolic) as structuring element $B_{d, \Delta}(\mathbf{x})$.

4.2. Practical implementation of bilateral flat morphology. Let us detail a simple approach to implement the bilateral flat operators using the stack principle. In practice, it can be assumed that image intensities of $f(\mathbf{x}) \in \mathcal{F}(\mathcal{T}, E)$ are numerical values which ranges in a closed subset of $L$ discrete values: $\mathcal{T}=\left\{t_{1}, t_{2}, \cdots, t_{L}\right\}$, with 
$\left(t_{l+1}-t_{l}\right)=\Delta t$. The thresholded set of $f$ at each $t_{l} \in \mathcal{T}$, defined by $X_{t_{l}}=\varpi_{t_{l}}(f)$, is called the cross-section or level-set at $t_{l}$ where $\varpi_{t}(f)$ is a mapping from $\mathcal{F}(E, \mathcal{T})$ to $\mathcal{P}(E)$ given by

$$
\varpi_{t}(f)=\{\mathbf{x} \in E \mid f(\{\mathbf{x}) \geq t\}
$$

The set of cross-sections constitutes a family of decreasing sets: $t_{\lambda} \geq t_{\mu} \Rightarrow X_{t_{\lambda}} \subseteq X_{t_{\mu}}$ and $X_{t_{\lambda}}=\cap\left\{X_{t_{\mu}}, \mu<\lambda\right\}$. Any image $f$ can be viewed as an unique stack of its cross-sections, which leads to the following supremal-based reconstruction property:

$$
f(\mathbf{x})=\sup \left\{t_{l} \mid \mathbf{x} \in X_{t_{l}}\right\}, \quad t_{l} \in \mathcal{T} \text {. }
$$

Or, alternatively [60] using a addition-based reconstruction formula:

$$
f(\mathbf{x})=\Delta t \sum_{l=1}^{L} \xi_{X_{t_{l}}}(\mathbf{x})
$$

where the characteristic function of set $X$, denoted $\xi_{X}: E \rightarrow\{0,1\}$, is defined by

$$
\forall X \in \mathcal{P}(E), \forall \mathbf{x} \in E, \quad \xi_{X}(\mathbf{x})= \begin{cases}1 & \text { if } \mathbf{x} \in X \\ 0 & \text { if } \mathbf{x} \in X^{c}\end{cases}
$$

It is well known in mathematical morphology that any binary increasing operator, such as the dilation and erosion, can be generalized to grey-level images by applying the binary operator to each cross-section, and then by reconstructing the corresponding grey-level image $[46,50]$. Consequently, for the bilateral flat dilation we can write

$$
\delta_{B_{d, \Delta}}(\mathbf{x})(f)(\mathbf{x})=\Delta t \sum_{l=1}^{L} \xi_{\delta_{B_{d, \Delta}(\mathbf{x})}\left(X_{t_{l}}\right)}(\mathbf{x})
$$

where $\delta_{B_{d, \Delta}(\mathbf{x})}\left(X_{t_{l}}\right)$ is the binary bilateral dilation defined by

$$
\delta_{B(\mathbf{x})}(X)=\cup\{B(\mathbf{x}) \mid \mathbf{x} \in X\} .
$$

Similarly, the bilateral flat erosion $\varepsilon_{B_{d, \Delta}}(\mathbf{x})(f)$, opening $\gamma_{B_{d, \Delta}}(\mathbf{x})(f)$ and closing $\varphi_{B_{d, \Delta}}(\mathbf{x})(f)$ can be implemented using the respective binary definition $[46,26]$.

Fig. 4.3 provides a comparison of bilateral flat dilation $\delta_{B_{d, \Delta}(\mathbf{x})}(f)(\mathbf{x})$ and bilateral flat closing $\varphi_{B_{d, \Delta}(\mathbf{x})}(f)(\mathbf{x})$, with $d=11$, of image $f(\mathbf{x})$ "Owl" with respect to different values of $\Delta$, and using the morphological Gaussian kernel (i.e., parabolic) for the spatially-variant structuring element. One can observe in both cases the excellent adaptive behavior of bilateral spatially-variant operators with respect to the standard spatially-invariant flat dilation $\delta_{B}(f)(\mathbf{x})$ and flat closing $\varphi_{B}(f)(\mathbf{x})$. For $\Delta=5$, the main structural edges are preserved but an effect of dilation or closing is produced in the regular zones. In the case of $\Delta=15$, a stronger morphological filtering is observed, but the basic structures are still preserved. For high values of critical contrast percentage, e.g., $\Delta=45$, the results obtained are already quite similar to those of the spatially-invariant operators.

Another comparison is given in Fig. 4.4, this time with bilateral flat erosion $\varepsilon_{B_{d, \Delta}(\mathbf{x})}(f)(\mathbf{x})$ and opening $\gamma_{B_{d, \Delta}(\mathbf{x})}(f)(\mathbf{x})$, using the same scale values of $d=11$ and 
$\Delta=5$, but considering the three cases of parabolic, conic and Log. Cauchy-Lorentz kernels in the thresholded structuring elements $B_{d, \delta}(\mathbf{x})$. The results for the three families of morphological kernels are roughly similar. But, once again, the logarithmic Cauchy-Lorentz is a good compromise between the kernels of first (conical) and second order (parabolic).

The last comparative example given in Fig. 4.5 deals with the problem of image denoising by means of bilateral approaches, using the (Log.) Cauchy-Lorentz kernel. We observe that the standard bilateral filtering $\Upsilon(f)\left(\mathbf{x} ; \widetilde{k}_{\alpha_{s}, \alpha_{i}}\right)$ produces a nice restoration, much better than the one obtained with bilateral flat median $M_{d, \Delta}(f)(\mathbf{x})$. In the framework of mathematical morphology, the two products of an opening and a closing yield an interesting operator, the averaged alternate filter, i.e.,

$$
\frac{\varphi_{B_{d, \Delta}(\mathbf{x})}\left(\gamma_{B_{d, \Delta}(\mathbf{x})}(f)\right)(\mathbf{x})+\gamma_{B_{d, \Delta}(\mathbf{x})}\left(\varphi_{B_{d, \Delta}(\mathbf{x})}(f)\right)(\mathbf{x})}{2},
$$

which presents skilful properties for denoising, since it combines a parallel simplification of bright and dark noisy structures. We remark in the example that the averaged alternate filter of diameter $d=7$ (Fig. 4.5-(f1)-bottom row, using bilateral flat opening/closing, produces a filtered image which is also quite satisfactory. In particular, the restored edges with the latter filter are better enhanced than with the standard bilateral filtering of equivalent size. This property of enhancement of structural edges is fundamentally intrinsic to the flat morphological operators (based on $\mathrm{max} / \mathrm{min}$ operations) with respect to the convolution-based operators (based on averaging operation). Nevertheless, it is well known that for Gaussian noise, like in the current example, convolution-based operators yields a better estimate than any combination of morphological ones. In the case of impulse noise or multiplicative noise, we can expect that bilateral morphological filters will lead to better denoising.

4.3. Applications. To complete the examples of this paper and more precisely, to motivate the interest of morphological bilateral filters for typical applications of mathematical morphology, we consider two applications. We discuss in both cases the effect of morphological bilateral flat operators in comparison with the standard ones.

The first application deals with the problem of contrast image enhancement using exclusively dilation and erosion. In fact, we propose to use the Kramer and Bruckner filter [32], which is known in the literature of mathematical morphology as toggle mapping [46, 50]. Basically, this nonlinear enhancement method consists in iterating a discrete operator that replaces every pixel intensity value by the dilation value or erosion value in the considered pixel neighborhood; the choice being determined by the closest one to the original pixel value, i.e.,

$$
\kappa_{B_{d, \Delta}}^{1-\text { iter }}(f)(\mathbf{x})=\left\{\begin{aligned}
\delta_{B_{d, \Delta}}(f)(\mathbf{x}) & \text { if }\left|\delta_{B_{d, \Delta}}(f)(\mathbf{x})-f(\mathbf{x})\right|<\left|f(\mathbf{x})-\varepsilon_{B_{d, \Delta}}(f)(\mathbf{x})\right| \\
\varepsilon_{B_{d, \Delta}}(f)(\mathbf{x}) & \text { if }\left|\delta_{B_{d, \Delta}}(f)(\mathbf{x})-f(\mathbf{x})\right|>\left|f(\mathbf{x})-\varepsilon_{B_{d, \Delta}}(f)(\mathbf{x})\right| \\
f(\mathbf{x}) & \text { if }\left|\delta_{B_{d, \Delta}}(f)(\mathbf{x})-f(\mathbf{x})\right|=\left|f(\mathbf{x})-\varepsilon_{B_{d, \Delta}}(f)(\mathbf{x})\right|
\end{aligned}\right.
$$

Fig. 4.6 gives a comparison of toggle mapping-based contrast enhancement between the standard spatially-invariant dilation/erosion and the bilateral counterparts. The (logarithmic) Cauchy-Lorentz kernel has been used for the bilateral cases. In the figure are given the first step and the results after three iterations. As we observe, the standard case using flat spatially-invariant dilation/erosion produces a strong enhancement which in some situations can lead to visual artefacts. Using bilateral flat dilation/erosion the enhancement is more gradual and involves less discretizationeffects. 
The second application considers the problem of image structure selection according to the morphology, which can be typically tackled using openings and closings. A first example is depicted in Fig. 4.7, which corresponds to the part of a radiography image of coronary vessels. By means of an opening, the bright structures of support size smaller than the structuring element are removed. Then, by computing the image difference between the original image and the opening image, the removed structures are enhanced. In the current example, the aims is to extract the vessel smaller than a given diameter which corresponds to Fig. 4.7(c) for the standard spatially-invariant opening and to Fig. 4.7(e) for the bilateral counterparts, using the (logarithmic) Cauchy-Lorentz kernel. As we can observe, for a comparable scale (size $d$ of structuring element), bilateral openings follows better the image structures and consequently, their residues lead to a better extraction vessels. In the last example of texture image given in Fig. 4.8, the purpose is to filter out the image in order to keep only the black structures of a diameter larger than a certain value. The example gives the results comparing the standard spatially-invariant opening/closing Fig. 4.8 (b)-(d) and the bilateral counterparts Fig. 4.8 (f)-(g), using again the (logarithmic) Cauchy-Lorentz kernel for the bilateral cases. The first opening is used to remove the bright lines smaller than the structuring element; the subsequent closing is applied to preserve only the black defect having a diameter larger than the structuring element, and the residue image gives the removed black defects. As we observe, the interest of the bilateral case here is twofold: on the one hand, the structures which are not removed preserve their contours; on the other hand, besides the "shape" of the target structure, its contrast is also considered to preserve it or not. Hence, only the well contrasted black spots of larger size are kept in the example.

4.4. Complexity. The complexity of a direct implementation for the computation of the bilateral structuring functions is $O(W \cdot N)$ where $W$ is the effective size of the spatial kernel and $N$ is the total number of pixels of the image. Typically, for the studied kernels, one can consider only the pixels in a square window of size $2 \eta_{s} \times 2 \eta_{s}$, and hence $W \propto\left(2 \eta_{s}\right)^{2}$. The complexity can be reduced to $O(\log W \cdot N)$ using a local histogram based algorithm [57]. In the case of flat morphology formulation using the stack implementation, the complexity should include the factor associated to the number of grey level, i.e., $O(W \cdot N \cdot L)$.

In order to compare with previous approaches, and according to the recent paper [23], the complexity of the morphological amoebas is $O\left(N \cdot n \omega^{2} \cdot \log \omega^{2}\right)$ and for the geodesic time-based neighbors is $O\left(N \cdot \omega^{2} \cdot \log \omega^{2}\right)$, where $n$ is the number of pixel graph connectivity and $\omega$ is the value of distance which defines the geodesic size. In both case, the implementation of the morphological operator using stack decomposition leads to complexities of $O\left(N \cdot L \cdot n \omega^{2} \cdot \log \omega^{2}\right)$ and $O\left(N \cdot L \cdot \omega^{2} \cdot \log \omega^{2}\right)$. The computational advantage of bilateral structuring functions is natural since they are based on the Euclidean distance whereas the amoebas are founded in geodesic distances. In addition, the complexity can still be reduced by using optimized algorithms for morphological filters [24]. 


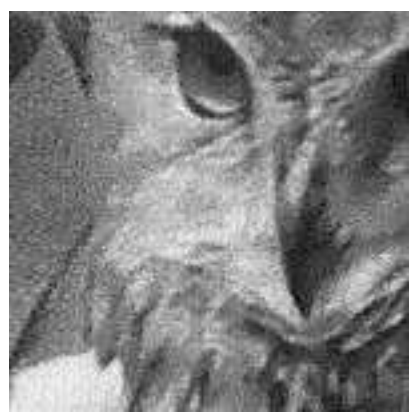

(a) Original

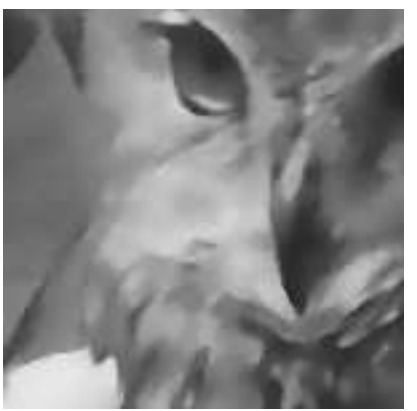

(b) Bilateral $\sigma_{s}=3, \sigma_{i}=0.1$ $P S N R=24.4$

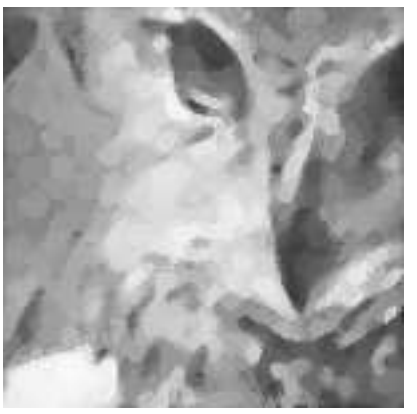

(c1) Bil. flat parab. dil. $d=11 ; \Delta=5$ $P S N R=16.0$

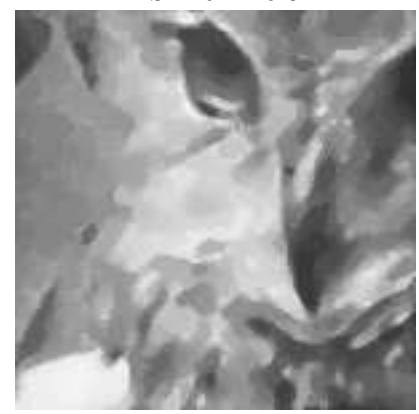

(d1) Bil. flat parab. clos. $d=11 ; \Delta=5$ $P S N R=19.2$

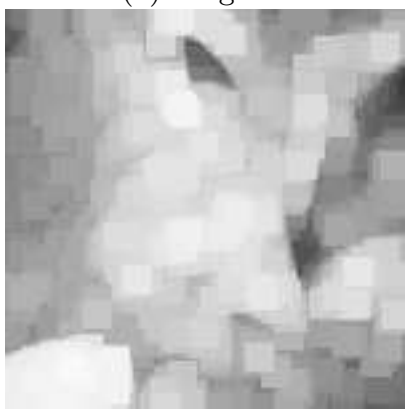

(c) Flat dil. $11 \times 11$ $P S N R=11.3$

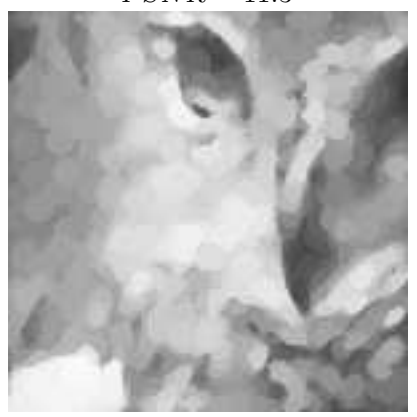

(c2) Bil. flat parab. dil. $d=11 ; \Delta=15$ $P S N R=14.0$

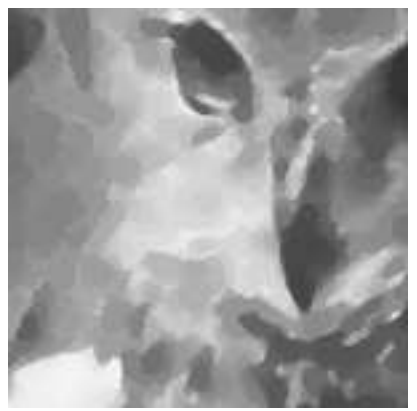

(d2) Bil. flat parab. clos. $d=11 ; \Delta=15$ $P S N R=18.1$

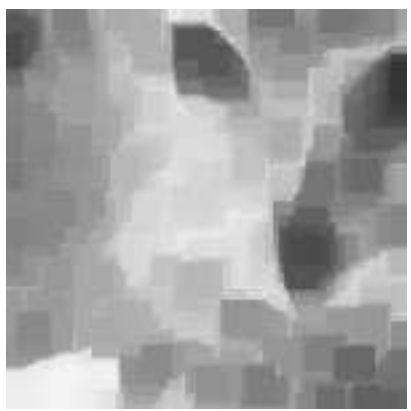

(d) Flat clos. $11 \times 11$ $P S N R=15.4$

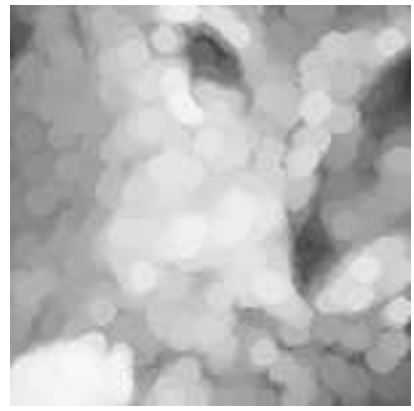

(c3) Bil. flat parab. dil. $d=11 ; \Delta=45$ $P S N R=12.3$

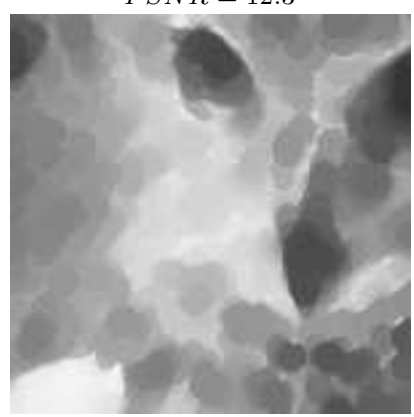

(d3) Bil. flat parab. clos. $d=11 ; \Delta=45$ $P S N R=16.7$

Figure 4.3. Comparison of bilateral flat dilation $\delta_{B_{d, \Delta}(\mathbf{x})}(f)(\mathbf{x})$ and bilateral flat closing $\varphi_{B_{d, \Delta}(\mathbf{x})}(f)(\mathbf{x})$, with $d=11$, of image $f(\mathbf{x})$ "Owl" with respect to different values of $\Delta$, and using the morphological Gaussian kernel (i.e., parabolic) for the spatially variant structuring element. The results can also be compared with the standard spatially invariant flat dilation $\delta_{B}(f)(\mathbf{x})$, flat closing $\varphi_{B}(f)(\mathbf{x})$ and bilateral filtering. 


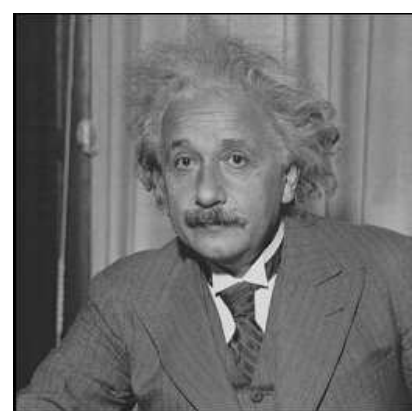

(a) Original

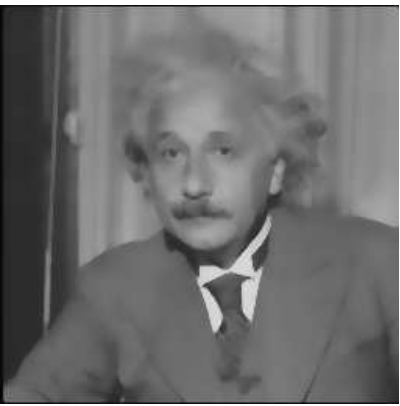

(b) Bilateral $\sigma_{s}=3, \sigma_{i}=0.1$

$P S N R=30.0$

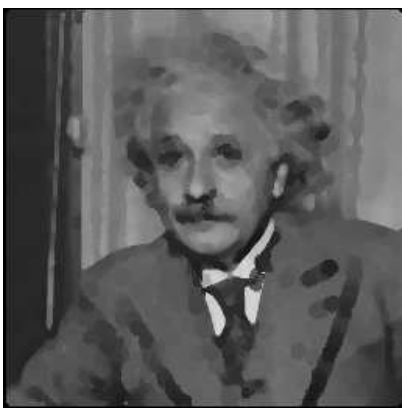

(c1) Bil. flat parab. eros.

$d=11 ; \Delta=5$

$P S N R=20.9$

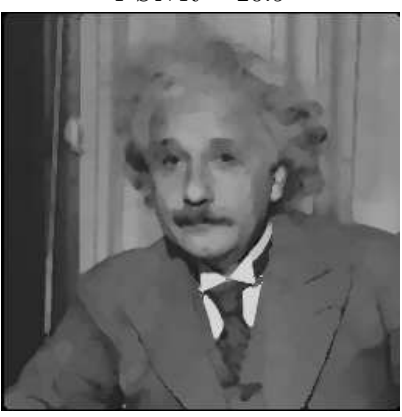

(d1) Bil. flat parab. open.

$$
d=11 ; \Delta=5
$$$$
P S N R=25.9
$$

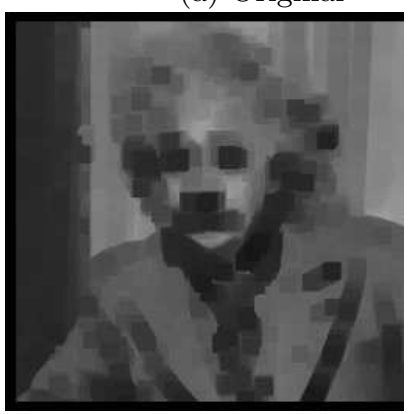

(c) Flat eros. $11 \times 11$ $P S N R=14.8$

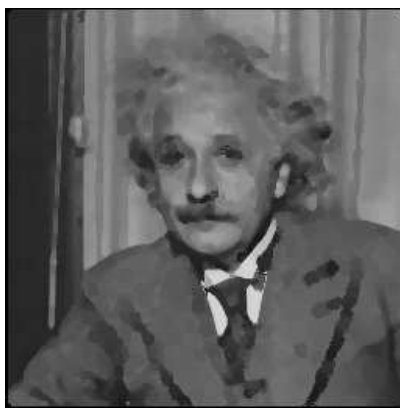

(c2) Bil. flat conic. eros.

$P S N R=22.3$

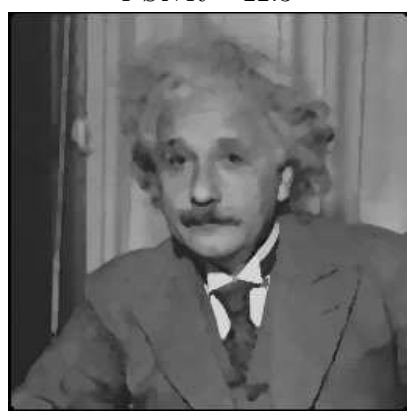

(d2) Bil. flat conic. open.

$$
\begin{aligned}
& d=11 ; \Delta=5 \\
& P S N R=26.8
\end{aligned}
$$$$
d=11 ; \Delta=5
$$

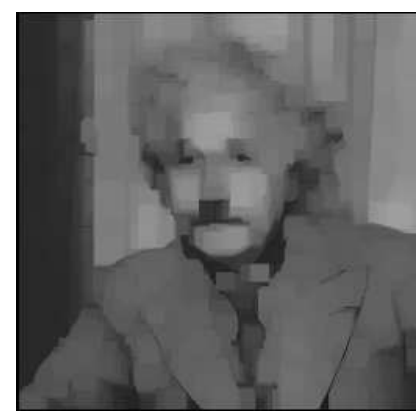

(d) Flat open. $11 \times 11$ $P S N R=20.6$

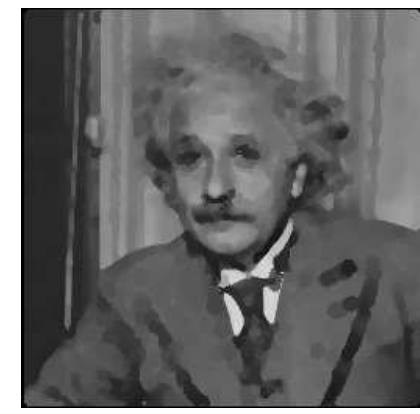

(c3) Bil. flat parab. Log-Cauc.-Lor. eros. $d=11 ; \Delta=5$ $P S N R=21.4$

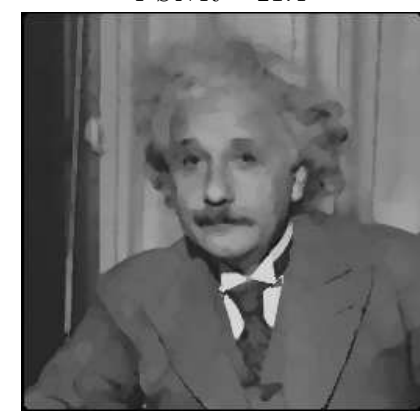

(d3) Bil. flat parab. Log-Cauc.-Lor. open.

$$
\begin{aligned}
& d=11 ; \Delta=5 \\
& P S N R=26.2
\end{aligned}
$$

Figure 4.4. Comparison of bilateral flat erosion $\varepsilon_{B_{d, \Delta}(\mathbf{x})}(f)(\mathbf{x})$ and bilateral flat opening $\gamma_{B_{d, \Delta}(\mathbf{x})}(f)(\mathbf{x})$, with $d=11$ and $\Delta=5$, of image $f(\mathbf{x})$ "Einstein" with respect to the three morphological kernels: (1) parabolic, (2) conic and (3) logarithmic Cauchy-Lorentz, used for for the spatially variant structuring element. The results can also be compared with the standard spatially invariant flat erosion $\varepsilon_{B}(f)(\mathbf{x})$, flat opening $\varphi_{B}(f)(\mathbf{x})$ and bilateral filtering using Gaussian kernel. 


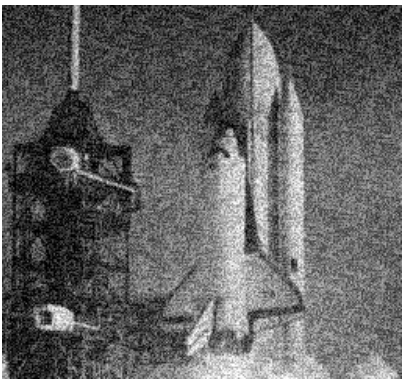

(a) Original

$P S N R=18.3$

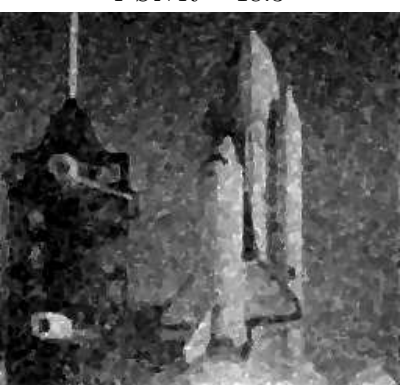

(d1) Bil. flat parab. open. clos. $d=4 ; \Delta=5$

$P S N R=20.0$

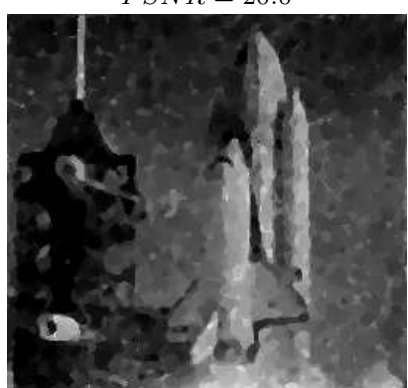

(d2) Bil. flat parab. open. clos.

$$
d=7 ; \Delta=5
$$

$P S N R=17.4$

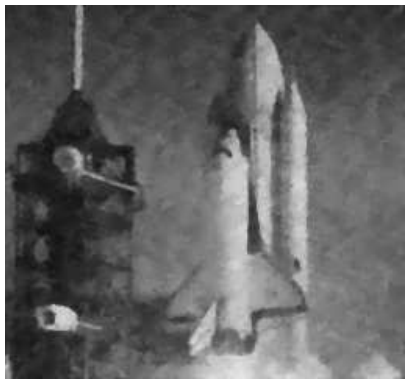

(b) Bilateral filt. $\alpha_{s}=3 ; \alpha_{i}=0.1$

$P S N R=27.5$

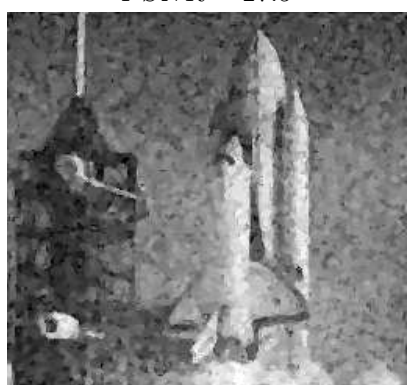

(e1) Bil. flat parab. clos. open. $d=4 ; \Delta=5$

$P S N R=19.8$

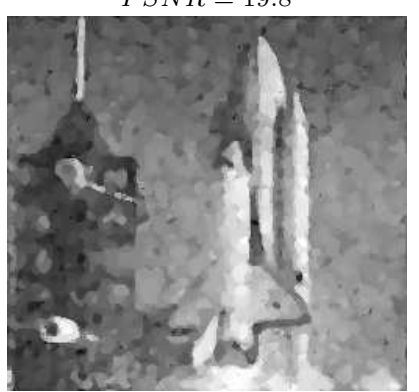

(e2) Bil. flat parab. clos. open.

$$
d=7 ; \Delta=5
$$$$
P S N R=17.3
$$

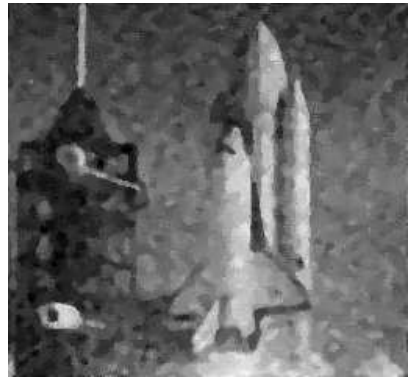

(c) Bil. flat median $d=7 ; \Delta=5$ $P S N R=24.9$

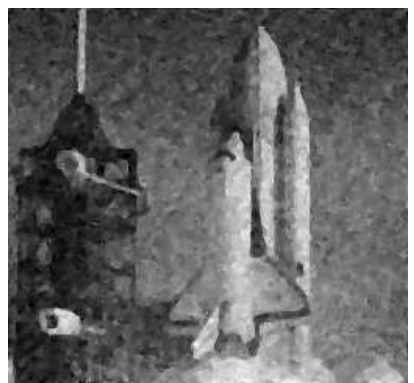

(f1) Averaged (d1) and (e1) $d=4 ; \Delta=5$

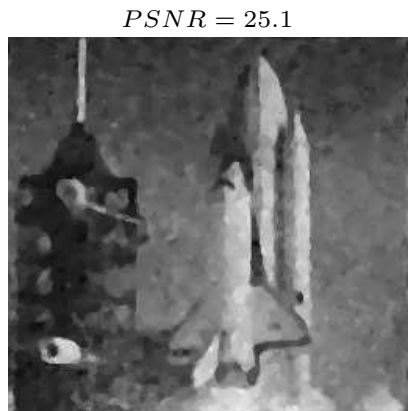

(f1) Averaged (d2) and (e2) $d=7 ; \Delta=5$ $P S N R=26.4$

FIGURE 4.5. Comparison of image denoising using bilateral approaches: (a) original noisy image $f(\mathbf{x})$, (b) bilateral filtering $\Upsilon(f)\left(\mathbf{x} ; \widetilde{k}_{\alpha_{s}, \alpha_{i}}\right)$, (c) bilateral flat median $M_{d, \Delta}(f)(\mathbf{x})$, (d) bilateral flat opening-closing $\varphi_{B_{d, \Delta}(\mathbf{x})}\left(\gamma_{B_{d, \Delta}(\mathbf{x})}(f)\right)(\mathbf{x})$, (e) bilateral flat closing-opening $\gamma_{B_{d, \Delta}(\mathbf{x})}\left(\varphi_{B_{d, \Delta}(\mathbf{x})}(f)\right)(\mathbf{x}),(f)$ averaged image of opening-closing and closing-opening. The (logarithmic) Cauchy-Lorentz kernel has been used for all the examples. 


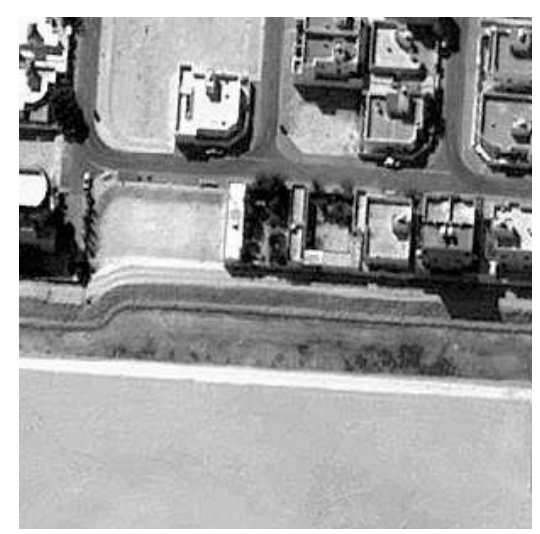

(a) Original

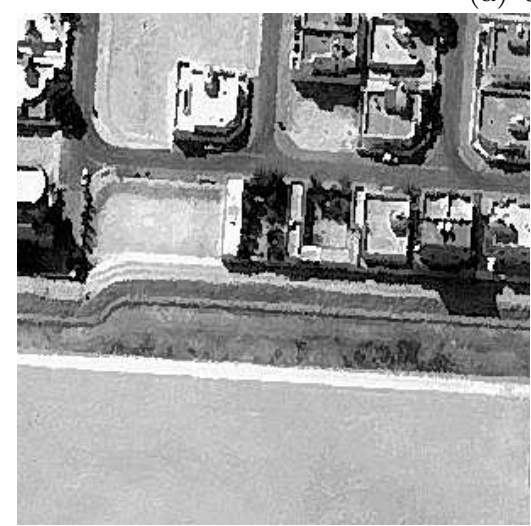

(b1) Flat Toggle-Mapping, 1 iter.

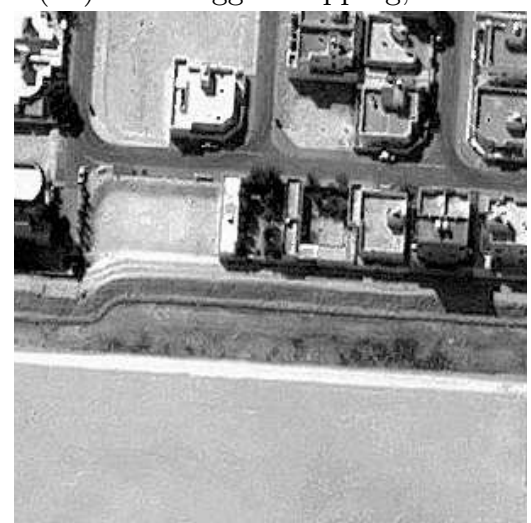

(c1) Bil. flat Toggle-Mapping, 1 iter. $d=3 ; \Delta=15$

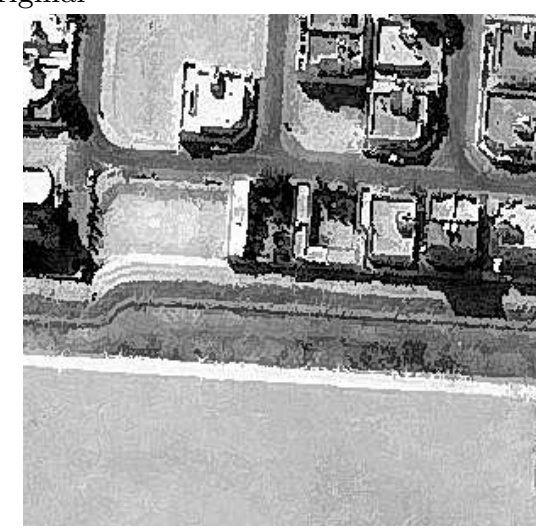

(b2) Flat Toggle-Mapping, 3 iter.

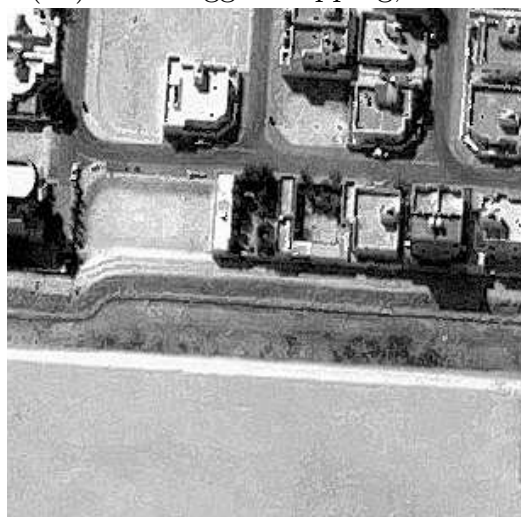

(c2) Bil flat Toggle-Mapping, 3 iter.

$$
d=3 ; \Delta=15
$$

FIGURE 4.6. Comparison of toggle mapping-based contrast enhancement $\kappa^{n-i t e r}(f)$ between the standard spatially-invariant dilation/erosion (b) and the bilateral counterparts (c). The (logarithmic) Cauchy-Lorentz kernel has been used for the bilateral cases. 


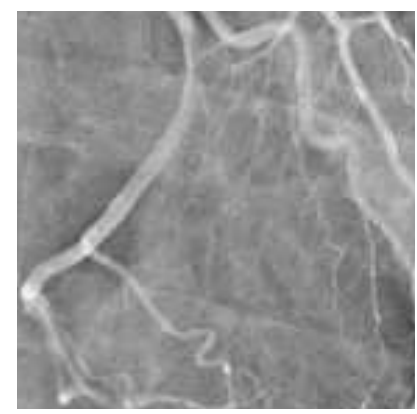

(a) Original $f$

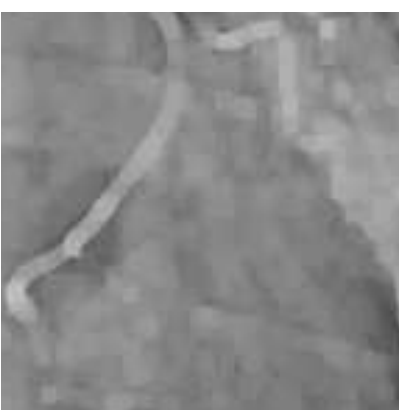

(b) $f_{1}=\gamma_{d}(f)$

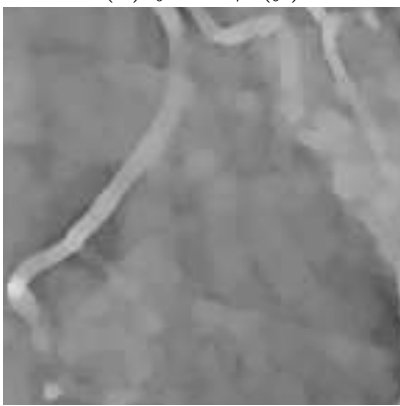

(d) $f_{3}=\gamma_{B_{d, \Delta}(\mathbf{x})}(f)$

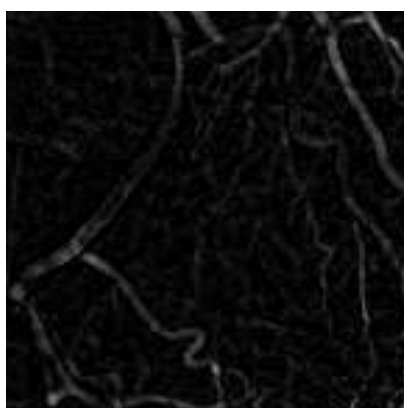

(c) $f_{2}=f-f_{1}$

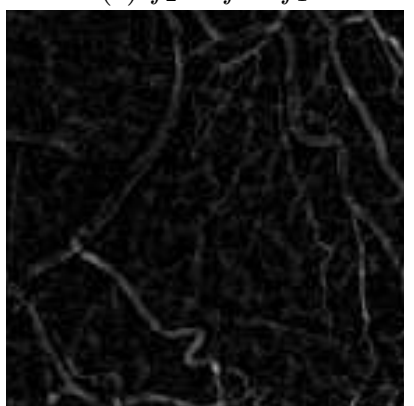

(e) $f_{4}=f-f_{3}$

Figure 4.7. Comparison of image structure extraction between the standard spatially-invariant openings (b)-(c) and the bilateral counterparts (d)-(e). The opening removes the bright structures smaller than the structuring element; the residue image gives the removed parts, i.e., coronary vessels. The (logarithmic) Cauchy-Lorentz kernel has been used for the bilateral operators. 


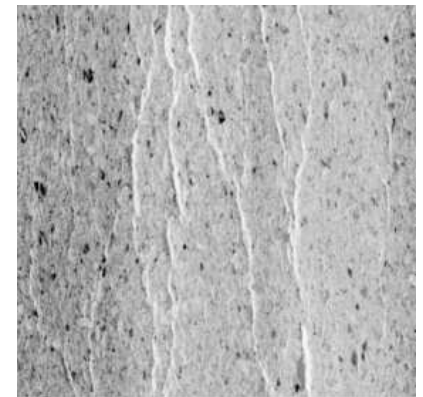

(a) Original $f$

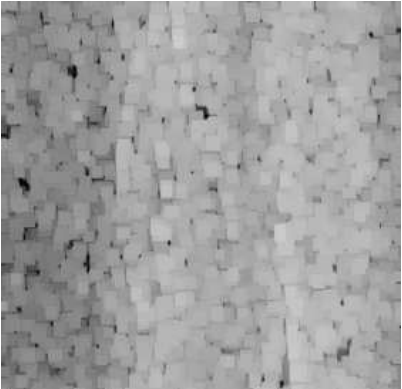

(b) $f_{1}=\gamma_{d}(f)$

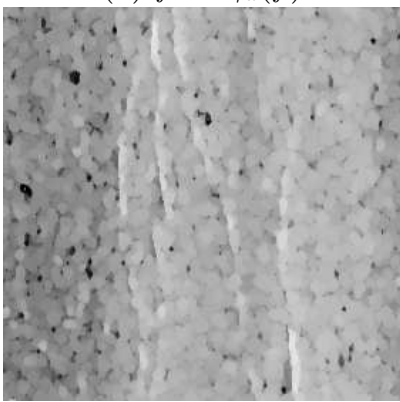

(d) $f_{3}=\gamma_{B_{d, \Delta}(\mathbf{x})}(f)$

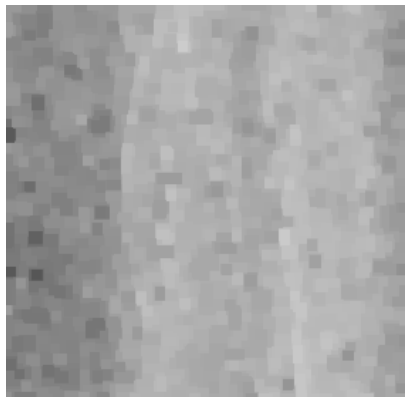

(c) $f_{2}=\varphi_{d}\left(f_{1}\right)$

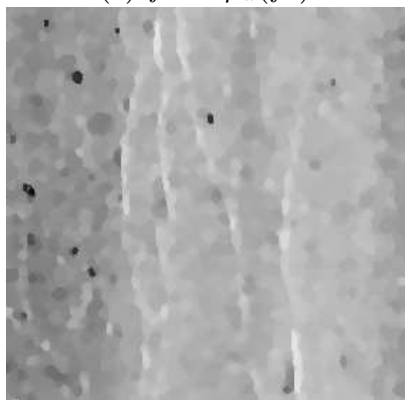

(e) $f_{4}=\varphi_{B_{d, \Delta}(\mathbf{x})}\left(f_{4}\right)$

FIGURE 4.8. Comparison of image structure filtering between the standard spatially-invariant opening/closing (b)-(c) and the bilateral counterparts (d)-(e). The first opening removes the bright lines smaller than the structuring element; the subsequent closing is used to preserve only the black defect having a diameter larger than the structuring element. The (logarithmic) Cauchy-Lorentz kernel has been used for the bilateral operators. 
5. Conclusions and perspectives. We have introduced in this paper the counterpart of bilateral filtering in the framework of mathematical morphology operators. Our starting point has been the notion of counter-harmonic filter, which provides a framework to explore the nonlinearization of convolution-based filters.

The main contributions of the paper can be summarized as follows.

- We have rediscovered the logarithmic connection between linear filters and the morphological ones, which allows us to propose the morphological equivalent to (locally adaptive) bilateral weighting convolution kernel: the bilateral structuring functions.

- We have considered, besides the Gaussian kernel, the use of Laplace and Cauchy-Lorentz kernels for bilateral filtering and the corresponding logarithmic counterparts to define bilateral structuring functions.

- We have introduced a low-complexity framework for flat spatially-variant filters using thresholded adaptive structuring functions and a theoretical appropriate formulation of operators.

The performance of the various nonlinear filters studied in the paper has been illustrated using a number of comparative examples, which showed their potential power in various image processing applications where morphology filters are successfully used.

As mentioned above, a key point of the methods discussed in the paper is their lowcomplexity in comparison with other adaptive morphological frameworks. In general, the optimized implementations require a fixed shape of the structuring element or, at least, a limited number of possible shapes. A possible solution to be explored is to define a limited library of possible spatially-variant structuring elements, describing the most common configurations for a particular size, then each shape obtained by thresholding the bilateral structuring functions can be approximated by one of the possible optimized configurations.

The preliminary results on non-local structuring functions will be developed in a future paper. The extension to multivariate images (such as multi/hyper-spectral images) will be considered in ongoing work. This requires in particular an appropriate formulation of vectorial morphology which is currently an active topic in mathematical morphology.

From a theoretical viewpoint, the continuation of the present work can follow two main directions. On the one hand, using the slope transform [17, 37] (closely related to the Legendre transform and the Young-Fenchel conjugate in convex analysis), an investigation about the properties of the logarithmic Cauchy-Lorentz functions, with respect to the parabolic families which are better known, and in particular on the behavior of the iteration of dilations/erosions using these non-standard functions can be achieved. On the other hand, the connection between the discrete operators proposed here and the morphological PDEs $[1,10,38]$ should be explored. In the literature they are already some works of PDE formulations of adaptive morphological operators $[39,14]$; however, a more deeper study is necessary to propose the morphological PDE which mimics the effects of the bilateral dilation/erosion.

\section{REFERENCES}

[1] L. Alvarez, F. Guichard, P. -L. Lions, And J. -M. Morel, Axioms and fundamental equations of image processing, Arch. for Rational Mechanics, 123 (1993), pp. 199-257. 
[2] J. Angulo, Pseudo-Morphological Image Diffusion using the Counter-Harmonic Paradigm, In Proc. of 2010 Advanced Concepts for Intelligent Vision Systems (ACIVS'2010), LNCS Vol. 6474, Part I, pp. 426-437, Springer, 2010.

[3] J. ANGUlo, Morphological bilateral filtering and spatially-variant adaptive structuring functions, In Proc. of 2011 International Symposium on Mathematical Morphology (ISMM'2011), LNCS Vol. 6671, pp. 212-223, Springer, 2011.

[4] S. Beucher, J.M. Blosseville, and F. LenoIr, Traffic Spatial Measurements Using Video Image Processing, Proc. of SPIE 848, pp. 648-655, 1987.

[5] M. Black, AND A. RANGARAJAn, On the unification of line processes, outlier rejection, and robust statistics with applications in early vision, International Journal of Computer Vision, 19 (1996), pp. 57-92,

[6] M. Black, G. Sapiro, D. H. Marimont, and D. Heeger, Robust anisotropic diffusion, IEEE Trans. on Image Processing, 7 (1998), pp. 421-432.

[7] R. VAn Den Boomgand, AND L. Dorst, The morphological equivalent of Gaussian scale-space, In Proc. of Gaussian Scale-Space Theory, pp. 203-220, Kluwer, 1997.

[8] R. VAN DEN BoomgaARd, AND J. VAN DE WEIJER, On the equivalence of local-mode finding, robust estimation and mean-shift analysis as used in early vision tasks, In Proc. of the 16th International Conference on Pattern Recognition (ICPR'02), Vol. 3, pp. 927-930, 2002.

[9] N. Bounynaya, AND D. Schonfeld, Theoretical foundations of spatially-variant mathematical morphology part ii: Gray-level images, IEEE Trans. Pattern Anal. Mach. Intell., 30 (2008), pp. 837-850.

[10] R. W. Brockett, and P. Maragos, Evolution equations for continuous-scale morphology, IEEE Trans. on Signal Processing, 42 (1994), pp. 3377-3386.

[11] A. Buades, B. Coll, And J. M. Morel, A review of image denoising methods, with a new one, Multiscale Modeling and Simulation, 4 (2006), pp. 490-530.

[12] P. S. Bullen, Handbook of Means and Their Inequalities, 2nd edition, Springer, 1987.

[13] B. Burgeth, and J. Weickert, An Explanation for the Logarithmic Connection between Linear and Morphological System Theory, International Journal of Computer Vision, 64 (2005), pp. 157-169.

[14] B. Burgeth, M. Breuss, L. Pizarro, and J. Weickert, PDE-driven adaptive morphology for matrix fields, In Proc. of Scale Space and Variational Methods in Computer Vision, LNCS Vol. 5567, pp. 247-258, Springer, Berlin, 2009.

[15] F. Catte, P. -L. Lions, J. -M. Morel, and T. Coll, Image selective smoothing and edge detection by nonlinear diffusion, SIAM Journal on Numerical Analysis, 29 (1992), pp. 182193.

[16] O. Cuisenaire, Locally adaptable mathematical morphology using distance transformations, Pattern Recognition, 39 (2006), pp. 405-416.

[17] L. Dorst, And R. VAn Den Boomgand, Morphological Signal Processing and the Slope Transform, Signal Processing, 38 (1994), pp. 79-98.

[18] M. ElaD, On the Origin of the Bilateral Filter and Ways to Improve It, IEEE Trans. on Image Processing, 11 (2002), pp. 1141-1151.

[19] D. Barash, A Fundamental Relationship between Bilateral Filtering, Adaptive Smoothing and the Nonlinear Diffusion Equation, IEEE Trans. on PAMI, 24 (2002), pp. 844-847.

[20] L. Florack, R. MaAs, and W. Niessen, Pseudo-Linear Scale-Space Theory, International Journal of Computer Vision, 31 (1999), pp. 1-13.

[21] J. M. Geusebroek, A. W. M. Smeulders, and J. van de Weijer, Fast anisotropic gauss filtering, IEEE Trans. on Image Processing, 12 (2003), pp. 938-943.

[22] R. C. Gonzalez, and R. E. Woods, Digital Image Processing, AAddison-Wesley, Boston, MA, USA, 1992.

[23] J. GrazZinI, AND P. SolLLe, Edge-preserving smoothing using a similarity measure in adaptive geodesic neighbourhoods, Pattern Recognition, 42 (2009), pp. 2306-2316.

[24] H. Hedberg, P. Dokladal, and V. Öwall, Binary Morphology with Spatially Variant Structuring Elements: Algorithm and Architecture, IEEE Transactions on Image Processing, 18 (2009), pp. 562-572.

[25] H. Heijmans, and C. Ronse, The Algebraic Basis of Mathematical Morphology. Part I: Dilations and Erosions, CVGIP: Image Understanding, 50 (1990), pp. 245-295.

[26] H.J.A.M. Heismans, Morphological Image Operators. Academic Press, Boston, 1994.

[27] A. D. Ioffe, and V. M. Tinomirov, Theory of extremal problems. North-Holland Publishing Company, 1979.

[28] P. T. JACKWAY, On Dimensionality in Multiscale Morphological Scale-Space with Elliptic Poweroid Structuring Functions, Journal of Visual Communication and Image Representation, 6(2) (1995), pp. 189-195. 
[29] P. T. Jackway, And M. Deriche, Scale-Space Properties of the Multiscale Morphological Dilation-Erosion, IEEE Trans. Pattern Anal. Mach. Intell., 18(1) (1996) pp. 38-51.

[30] R. Kimmel, R. Malladi, And N. Sochen, Images as Embedded Maps and Minimal Surfaces: Movies, Color, Texture, and Volumetric Medical Images, International Journal of Computer Vision, 39(2) (2000) pp. 111-129.

[31] G. A. Korn, And T. M. Korn, Mathematical Handbook for Scientists and Engineers: Definitions, Theorems, and Formulas for Reference and Review, McGraw-Hill, New York, 1968.

[32] H. P. KRAMER, AND J. B. BRUCKNER, Iterations of a non-linear transformation for enhancement of digital images, Pattern Recognition, 7 (1975) pp. 53-58.

[33] R. L. Lagendijk, J. Biemond, And D. E. Boekee, Regularized iterative image restoration with ringing reduction, IEEE Trans. Acoust., Speech, Signal Processing, 36 (1988), pp. 18741887.

[34] J. S. LEE, Digital image enhancement and noise filtering by use of local statistics, IEEE Trans. PAMI, 2 (1980), pp. 165-168.

[35] J. S. LEE, Digital image smoothing and the sigma filter, Computer Vision, Graphics and Image Processing, 24 (1983), pp. 255-269.

[36] R. Lerallut, E. Decenciere, And F. Meyer, Image Filtering Using Morphological Amoebas, Image Vision and Computing, 25 (2007), pp. 395-404.

[37] P. Maragos, Slope Transforms: Theory and Application to Nonlinear Signal Processing, IEEE Trans. on Signal Processing, 43 (1995), pp. 864-877.

[38] - Differential morphology and image processing, IEEE Transactions on Image Processing, 5 (1996), pp. 922-937.

[39] P. Maragos, And C. VAchier, A PDE Formulation for Viscous Morphological Operators with Extensions to Intensity-Adaptive Operators, In Proc. of 15th IEEE international conference on Image processing (ICIP'08), pp. 2200-2203, 2008.

[40] P. Maragos, And C. VAChIER, Overview of adaptive morphology: trends and perspectives, In Proc. of 16th IEEE international conference on Image processing (ICIP'09), pp. 2241-2244, 2009.

[41] P. Perona, and J. Malik, Scale-Space and Edge Detection Using Anisotropic Diffusion, IEEE Trans. Pattern Anal. Mach. Intell., 12(1990), pp. 629-639.

[42] S. Paris, P. Kornprobst, J. Tumblin, and F. Durand, Bilateral Filtering: Theory and Applications, Foundations and Trends in Computer Graphics and Vision, 4 (2008), pp. 173.

[43] J. Roerdink, Adaptivity and group invariance in mathematical morphology, In Proc. of 16th IEEE international conference on Image processing (ICIP'09), pp. 2253-2256, 2009.

[44] R. T. Rockafellar, Convex Analysis, Princeton University Press, Princeton, 1970.

[45] J. Serra, Image Analysis and Mathematical Morphology, Academic Press, London, 1982.

[46] J. Serra, Image Analysis and Mathematical Morphology. Vol II: Theoretical Advances, Academic Press, London, 1988.

[47] N. Sochen, R. Kimmel, And R. Malladi, A general framework for low level vision, IEEE Trans. on Image Processing, 7 (1998), pp. 310-318.

[48] N. Sochen, R. Kimmel, And A. M. BRUCKstein, Diffusions and confusions in signal and image processing, Journal of Mathematical Imaging and Vision, 14 (2001), pp. 195-209.

[49] P. Sollle, Generalized geodesy via geodesic time, Pattern Recognition Letters, 15 (1994), pp. 1235-1240.

[50] - Morphological Image Analysis, Springer-Verlag, Berlin, 1999.

[51] S.R. Sternberg, Grayscale morphology, Comput. Vision Graphics Image Process., 35(3) (1986), pp. 333-355, .

[52] C. Tomasi, and R. Manduchi, Bilateral filtering for gray and color images, In Proc. of 6th Int. Conf. Computer Vision (ICCV'98), pp. 839-846, 1998.

[53] L. J. VAN VLIET, Robust Local Max-Min Filters by Normalized Power-Weighted Filtering, In Proc. of IEEE 17th International Conference of the Pattern Recognition (ICPR'04), Vol. 1, pp. 696-699, 2004.

[54] C. Vachier, And F. Meyer, News from Viscousland, Proc. of Inter. Symp. on Mathematical Morphology (ISMM'07), pp. 189-200, 2007.

[55] R. Verdú-Monedero, J. Angulo And J. Serra, Anisotropic morphological filters with spatially-variant structuring elements based on image-dependent gradient fields, IEEE Trans. on Image Processing, 20 (2011), pp. 200-212.

[56] J. WeIckert, Anisotropic Diffusion in Image Processing, ECMI Series, Teubner-Verlag, Stuttgart, Germany, 1998.

[57] B. WeIss, Fast median and bilateral filtering, ACM Transactions on Graphics, 25 (2006), 
pp. 519-526.

[58] M. Welk, Families of generalised morphological scale spaces, In Proc. of 4th Inter. Conf. of Scale-Space Methods in Computer Vision, LNCS Vol. 2695, pp. 770-784, Springer, 2003.

[59] M. Welk, M. Breuss, And O. Vogel, Morphological amoebas are self-snakes, Journal of Mathematical Imaging and Vision, 39 (2011), pp. 87-99.

[60] P.D. Wendt, E.J. Coyle, and N.C. Gallagher, Stack Filters, IEEE Trans. on Acoustics, Speech, and Signal Processing, 34 (1986), pp. 898-911.

[61] G. Z. Yang, P. Burger, D. N. Firmin, and S. R. Underwood, Structure adaptive anisotropic image filtering, Image and Vision Computing, 14 (1996), pp. 135-145.

[62] L. Yaroslavsky, Digital Picture Processing, An Introduction, Springer Verlag, Berlin, Heidelberg, 1985. 